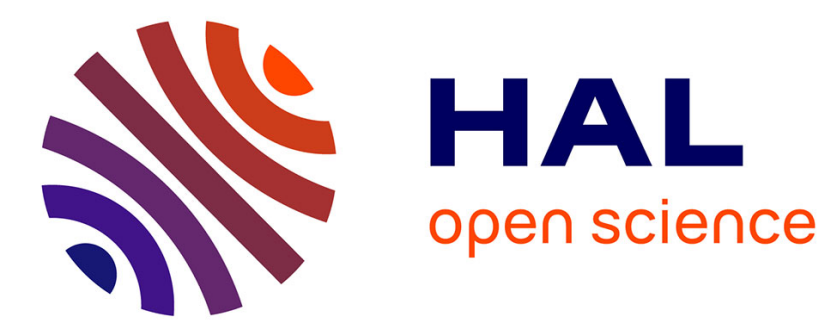

\title{
SISO model-based control of separated flows: Sliding mode and optimal control approaches
}

Maxime Feingesicht, Andrey Polyakov, Franck Kerhervé, Jean-Pierre Richard

\section{To cite this version:}

Maxime Feingesicht, Andrey Polyakov, Franck Kerhervé, Jean-Pierre Richard. SISO model-based control of separated flows: Sliding mode and optimal control approaches. International Journal of Robust and Nonlinear Control, 2017, 10.1002/rnc.3843 . hal-01587824

\section{HAL Id: hal-01587824 \\ https://hal.inria.fr/hal-01587824}

Submitted on 14 Sep 2017

HAL is a multi-disciplinary open access archive for the deposit and dissemination of scientific research documents, whether they are published or not. The documents may come from teaching and research institutions in France or abroad, or from public or private research centers.
L'archive ouverte pluridisciplinaire HAL, est destinée au dépôt et à la diffusion de documents scientifiques de niveau recherche, publiés ou non, émanant des établissements d'enseignement et de recherche français ou étrangers, des laboratoires publics ou privés. 


\title{
SISO model-based control of separated flows: Sliding mode and optimal control approaches
}

\author{
Maxime Feingesicht ${ }^{1,2,3}$, Andrey Polyakov ${ }^{1,2}$, Franck Kerhervé ${ }^{4}$, and \\ Jean-Pierre Richard ${ }^{1,2,3}$ \\ ${ }^{1}$ Inria Lille Nord Europe, Parc scientifique de la Haute Borne, 40 \\ Avenue du Halley Bat. A, Park Plaza, 59650 Villeneuve-d'Ascq, France \\ ${ }^{2}$ CRIStAL UMR CNRS 9189, Ecole Centrale de Lille, BP48, Cité \\ Scientifique, 59651 Villeneuve d'Ascq, France \\ ${ }^{3}$ Ecole Centrale de Lille, Cit Scientifique, 59651 Villeneuve-d'Ascq, \\ France \\ ${ }^{4}$ Institut P', CNRS UPR 3346, Universit de Poitiers, 11 Boulevard \\ Marie et Pierre Curie, BP 30179, F86962 Futuroscope Chasseneuil \\ Cedex
}

September 14, 2017

\begin{abstract}
The challenging problem of active control of separated flows is tackled in the present paper using model-based design principles, and applied to data issued from a two-dimensional separated flow experiment. First, a bilinear state and input delay model of the system has been obtained from experimental data by means of a modified identification procedure. Adequacy and precision of the obtained model are demonstrated and compared with existing results. Next, two control problems (setpoint tracking and optimal control) have been formulated and studied using sliding mode control methodology and averaging analysis. The theoretical control results are supported with numerical simulations.
\end{abstract}

\section{Introduction}

Control problems for separated turbulent flow are of great interest in the view of many modern challenges [1]. For example, aerodynamic losses are believed to be one of the main source of energy wastage for a vehicle at speeds higher than $50 \mathrm{~km} / \mathrm{h}$. According to the existing ecological estimates, the reduction of these losses at $25 \%$ will decrease pollution for more than $10^{7}$ tonnes of $\mathrm{CO}_{2}$ per year. Optimization of the vehicles shapes remain the preferred solution for reducing aerodynamic losses (or drag) due to turbulence related mechanisms such as flow separation. Such optimization constitutes a passive control approach which can not lead to an optimal solution, in particular when the incoming flow faces dramatic changes due to large unsteady events. Other passive control solutions include fixed objects such as small deltawings or so-called vortex generators located on the vehicle surface in order to manipulate the boundary layer (see [2] and [3] for extensive reviews). Such strategy requires generally extensive 
parametric studies to find the geometrical parameters (dimensions and locations) of the actuators leading to a solution which can only be optimal for a limited range of flow conditions. In such a context, active control strategies [1], [4], [5], [6], [7], [8] implying actuators interacting with the flow and whose control parameters can be varied on-line to maintain an optimal solution leading to minimization of the aerodynamic losses constitutes an attractive solution.

Fluidic actuators are the most encountered solutions in turbulent flow control applications, at least at the academic level (see, e.g. [9], [10], [11]). Flow separation occurring due to a large adverse pressure gradient such as observed for two-dimensional flap (see [12], [13], [14], [15], [16]) constitutes one of the standard benchmark for development of new actuators or advanced control strategies [4], [5], [17], [18] and [19]. This configuration will be considered in the present paper as a test case to apply recently developed methods of the Control Theory.

The main difficulty of turbulence control is that the behavior of the physics underlying the plant (here designing the flow system) is highly nonlinear. The flow dynamics are effectively driven by the Navier-Stokes equation which is a distributed parameter model. Implementation of controllers or observers for such infinite dimensional systems needs huge computational resources which cannot be provided in real-time (see [20], [21], [22]).

Various strategies for separated flow control can be found in the literature. The most of them use very local (linear) models [6], [7], [8] (i.e. basically skip nonlinear turbulent dynamics) and deal mainly with feedforward control [4], [5], [17], [18] and [19]. In this context, the recent control design approach based on machine learning (model-free) techniques [23] looks rather promising among others. However, long series of repeated experiments are required for its proper tuning. In addition, the machine learning approach leaves open a question of robustness of the designed control and the convergence is not totally proven. A recent survey about various approaches to flow control design is given by [1]. Model-based robust control of separated flows remains of particular interest and can be implemented in real system without too much complexity. One of the objectives of the present paper is to study new perspectives in this topic.

To design a practically realizable control law, a sufficiently simple model of the plant should be constructed. For this purpose the paper uses a modified grey-box identification technique and constructs a model that adequately describes an input/output behavior of the flow control system. The obtained model is bilinear and presented by difference-differential equation with state/input delays. One more feature of the control system under consideration is relay ("ON"/"OFF") actuation provided by pulsed jets (air blowers). The preliminary results on modeling of the control system of separated flows can be found in [24].

The present paper is focused on development of control strategies by means of applying appropriate mathematical methods to the obtained bilinear time-delay model, so its main contribution is theoretical. In particular, an optimal control problem is formulated and a scheme for a sub-optimal feedforward periodic control design is developed based on averaging analysis. Next, a setpoint tracking algorithm is designed using a sliding mode control methodology [25], [26], [27], [28], [29] jointly with a prediction technique (see, e.g. [30], [31]). The choice of sliding mode control technique is motivated by the nature of the actuators used in the experiments which can only be turned on or off, such as relays. It is well known [25] that the sliding mode is the usual operation mode of relay control systems. Relay control and/or sliding mode control systems with delays are studied, for example, in [32], [33]. Despite of the fact that bilinear systems were considered in literature (see, e.g. [34]), to the best of our 
knowledge, the considered control problems for bilinear models with state and input delays has never been studied before.

Notation:

- $\mathbb{R}$ is the set of real numbers, $\mathbb{R}_{+}=\{x \in \mathbb{R} \mid x \geq 0\}$;

- $\mathbf{C}_{\Omega}$ is the space of continuous functions;

- $\mathbb{1} \in \mathbf{C}_{\Omega}$ is the unit constant function: $\mathbb{1}(s)=1, \forall s \in \Omega$;

- $\mathbf{L}_{\Omega}^{2}$ is the space of quadratically integrable functions, $\|z\|_{\mathbf{L}_{\Omega}^{2}}=\sqrt{\int_{\Omega} z^{2}(s) d s}$;

- $\mathbf{L}_{\Omega}^{\infty}$ is the space of locally measurable essentially bounded functions, $\|z\|_{L^{\infty}}=$ $\underset{s \in \Omega}{\operatorname{ess} \sup }|z(s)|$;

- if $\tau>0, y \in \mathbf{L}_{\mathbb{R}}^{\infty}$ and $t \in \mathbb{R}$ then $\xi_{\tau}(t) \in \mathbf{L}_{[-\tau, 0]}^{\infty}:\left(y_{\tau}(t)\right)(\sigma)=y(t+\sigma)$ for $\sigma \in$ $[-\tau, 0]$. The notation $y_{\tau}(t)$ and $y_{-\tau}(t)$ is commonly used for time-delay models $[35]$.

\section{Flow Control System}

\subsection{Flow Control Problem}

The problem of flow control is basically the meeting point of three research areas [1] :

1. Fluid Mechanics (for analysis of flow dynamics and formulation of control goals),

2. Electronics (for sensing and actuation developments),

3. Control Theory and Optimization (for designing of control laws).

Flow control experimental setup is generally designed and assembled based on current technological achievements in the field of fluid dynamics and electrical engineering. In such a context, the operator cannot have any impact on the set-up, except on the control parameters which drive the actuators. The problem resulting is therefore to optimize efficiency and robustness of the controller by designing appropriate control algorithms.

To the best of our knowledge, the paper presents the first attempts in the context of non-linear (in particular, bilinear) SISO model-based control design for separated flows. It deals with two classical control paradigms: open-loop (feedforward) and closed-loop (feedback). While an optimal control problem is studied in the open-loop context, sliding mode methodology is applied in order to design robust feedback which tracks a given setpoint.

\subsection{Experimental test case}

The experimental test case considered is that of a turbulent boundary layer flow occurring separation along a two-dimensional ramp whose geometry and dimensions are illustrated in Fig. 1. Full details of the experiments, which were conducted in the large boundary-layer wind tunnnel at Laboratoire de Mécanique de Lille (France) can be found in [13], [36]. The boundary layer flow first develops along a flat horizontal plate (floor of the wind-tunnel) before reaching a smooth convergent where it occurs acceleration. The flow continues to develop along a slightly inclined flat plate to 
recover a zero pressure streamwise gradient. This is followed by a flap along which the boundary layer occurs separation and reattaches further downstream to the floor of the wind-tunnel. This is illustrated in Fig. $2 \mathrm{~b}$ where streamlines for the averaged natural flow are reported. Note that the flow comes from the left of the figure. The ramp geometry is shown as the thick black line. In the present configuration, the location where the flow separates from the wall is fixed and located at the edge between the inclined flat plate and the flap (chosen as origin of the coordinate system in figure Fig. 2). Just downstream of the edge, a shear layer forms and a recirculation region (reversed flow) appears along the flap due to flow separation. The border between positive and negative streamwise mean velocity is represented as the blue line. Below this blue line, the flow is, in average, reversed compared to the flow above the line. The flow in this separation region constitutes the physical system of interest and to control, the main objective of the control being to reduce the recirculation region.

An array of 22 co-rotating round jets, air blowers, aligned parallel to the flap edge is used as actuators. The control input u(t) is a relay ("on" /"off") signal sent to the actuators with a given frequency and duty cycle. An example of the averaged flow obtained when using continuous actuation (relay remains "ON") is illustrated in Fig. 2a. Compared to the natural flow discussed previously and shown in Fig. 2b, the region of reversed flow is drastically reduced and the flow is found to be almost fully attached to the bottom wall.

For real-time survey, hot-film sensors located along the flap are used to measure the gain in skin friction: an increase in friction gain being representative of flow reattachement. In the present configuration, output voltages of hot-film sensors are the only signals that can be measured in on-line and utilized for control proposes. The output voltages of the sensors are constants in the steady state. From the point of view of Control Theory, the control problem examined here admits conventional interpretation given in Fig. 3.

\subsection{Control Aims}

\subsubsection{Setpoint Tracking Control}

Based on flow velocity surveys (not detailed in the present paper), the capability of the actuators to reattach the flow to the wall has been shown by Raibaudo [37], [36] to be well characterized by skin friction gain measured by the hot-film sensors. Therefore, the first control problem to be studied here is stabilization of the output $y$ at the desired setpoint $y^{*}$. The relay nature of actuators motivates us to apply sliding mode principles in order to design a robust feedback law, which guarantees $y(t) \rightarrow y^{*}$ as $t \rightarrow \infty$.

\subsubsection{Optimal Control}

Let us consider the cost functional

$$
J(y, u)=\lim _{T \rightarrow+\infty} \frac{1}{T} \int_{0}^{T} \alpha y(s)-(1-\alpha) u(s) d s
$$

with $0 \leq \alpha \leq 1$, which characterizes the averaged value of $y$ in the steady state and the averaged control value required to obtain it. Since increasing of the output $y$ implies better reduction of turbulence [12] and our objective is to reattach the flow as much as possible we also study the problem: to design a control law $u$ such that

$$
J(y, u) \rightarrow \max
$$




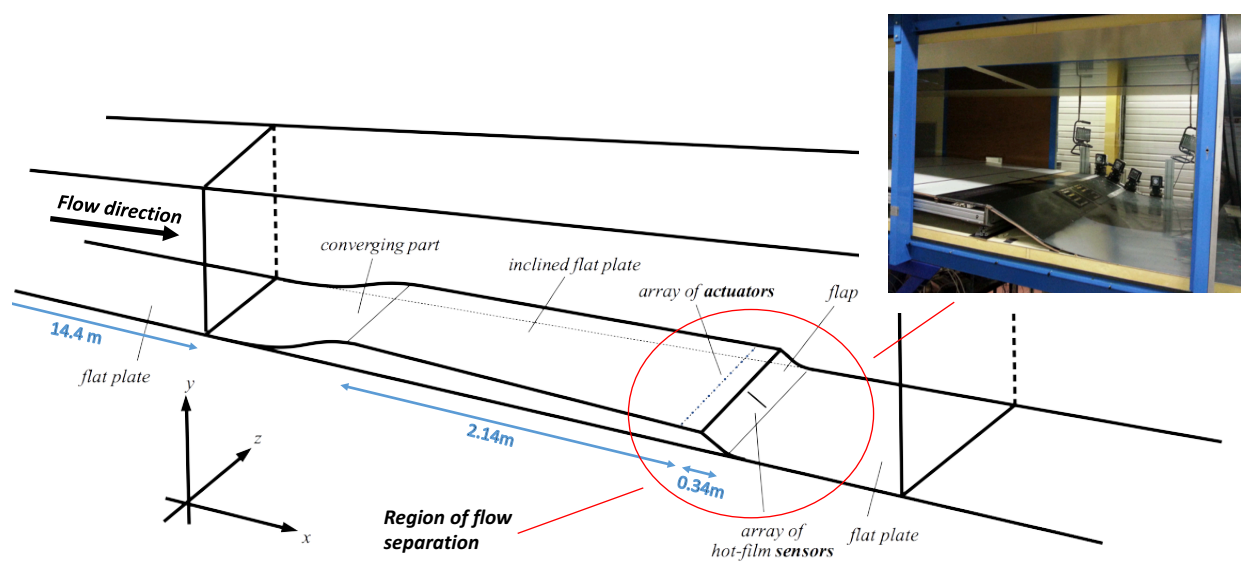

Figure 1: Diagram and photo of the experimental setup Courtesy of Laboratoire de Mécanique de Lille

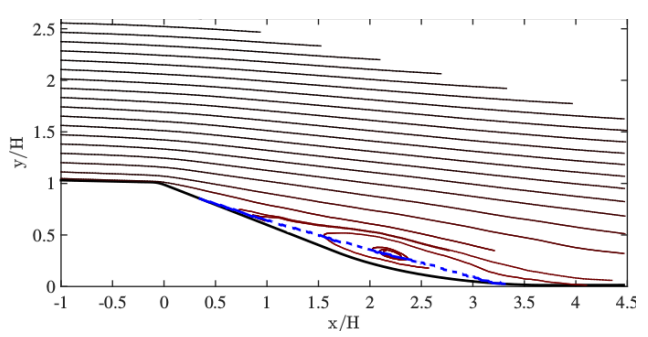

(a)

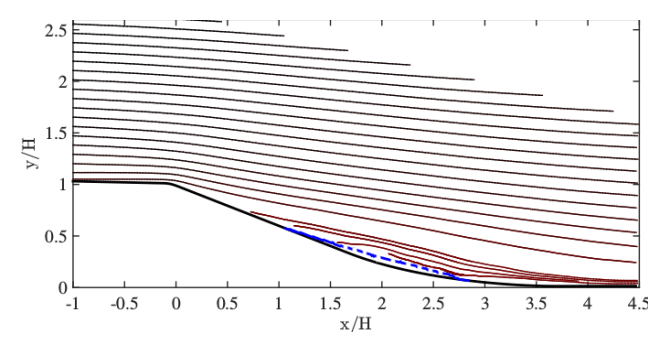

(b)

Figure 2: Streamlines for (a) the natural flow without control and (b) the flow under continuous actuation. The blue line represents the border between the reversed flow (negative streamwise velocity, region of the flow below the line) and the freestream (positive streamwise velocity, region of the flow above the line). In the controlled case (b) the recirculation region is shown to be drastically reduced and the flow almost fully reattached to the wall.

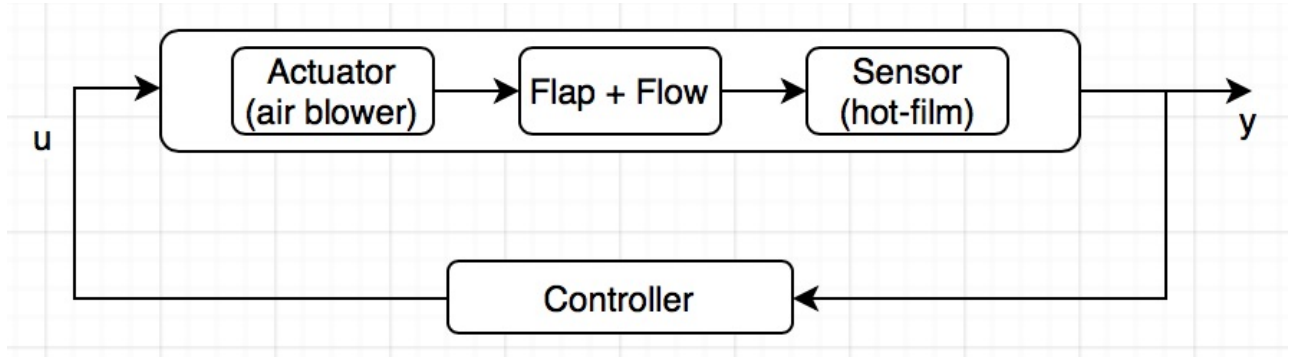

Figure 3: Feedback scheme 
The trade-off between the turbulence reduction and the energy consumed by the actuation is provided by the choice of the parameter $\alpha$.

\section{Input-Output Modeling of Flow Separation}

\subsection{Experimental Data and Pre-Processing}

The only data we can use for modeling are the input signals to the actuators and the output voltages of the hot-film sensors measured with a frequency of $1 \mathrm{kHz}$. Therefore, we cannot design model separately for actuator, sensor and plant, but our model will implicitly include them all.

Several experiments have been done in order to collect an experimental database appropriate for model design. Each experiment consists of two phases: actuation and relaxation. Actuation is done by means of a periodic on/off input signal $u$ with a fixed frequency and duty circle (DC). Actuation time is 5 seconds. Seven different input signals have been tested: 1) constant input; 2) Freq $=4 \mathrm{~Hz}$ with $\mathrm{DC}=50 \%$; 3) Freq $=4 \mathrm{~Hz}$ with $\mathrm{DC}=80 \%$; 4) Freq $=8 \mathrm{~Hz}$ with $\mathrm{DC}=50 \%$; 5) Freq $=8 \mathrm{~Hz}$ with $\mathrm{DC}=80 \%$; 6) Freq $=80 \mathrm{~Hz}$ with $\mathrm{DC}=50 \%$; 6) Freq $=80 \mathrm{~Hz}$ with $\mathrm{DC}=80 \%$.

During the relaxation phase the control is switched off for 5 seconds in order to let the flow to return to a natural steady separated state. Each experiment is repeated for more than 50 times and the results are phase averaged in order to obtain an output signal less effected by measurement noises and exogenous perturbations. This phase-averaged data (see, Fig. 4) is utilized for modeling.

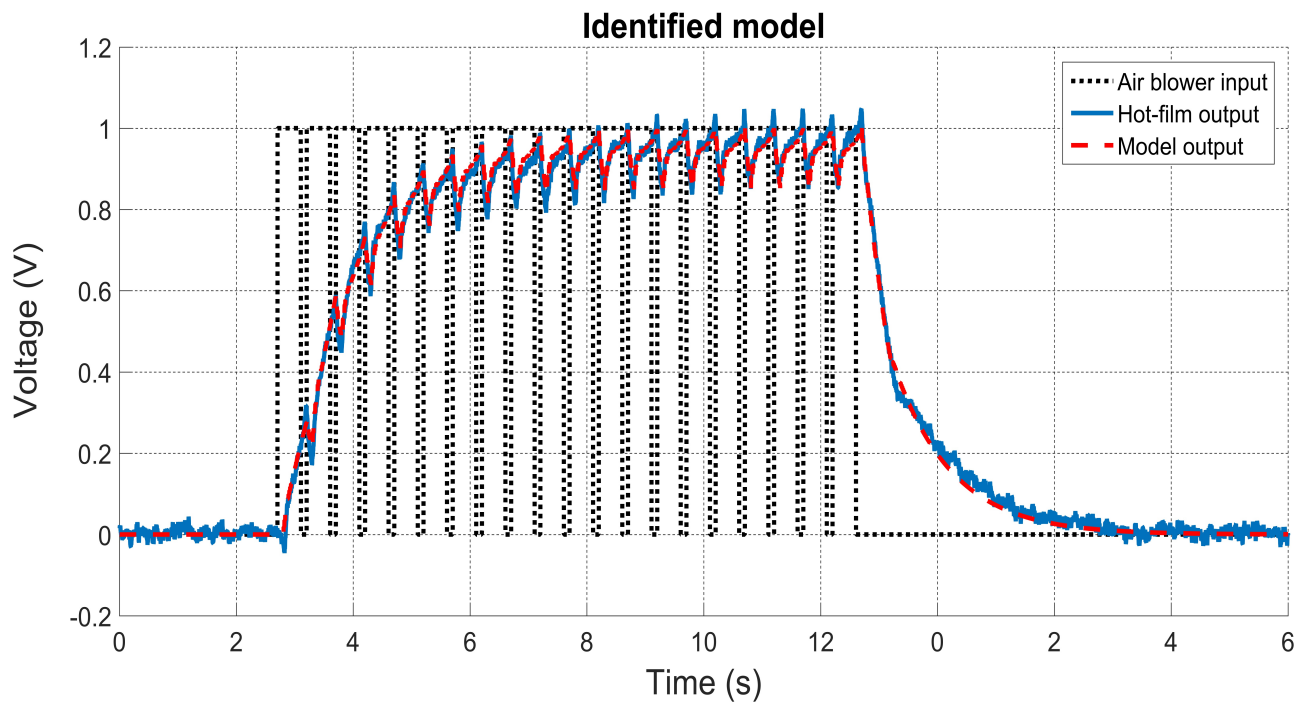

Figure 4: Phase-averaged data for Freq $=4 \mathrm{~Hz}, \mathrm{DC}=80 \%$

\subsection{Bilinear Model}

The dynamics of the flow considered here are highly nonlinear and governed by partial differential equations (e.g. Navier-Stokes equations). The only SISO (Single Input Single Output) model can be designed using the experimental dataset. However, this model should take into account nonlinearity and an infinite dimensional nature of the control system. That is why we identify an appropriate model from the class 
of bilinear control systems governed by differential equations with time delays (i.e. differential-difference equations) :

$$
\dot{y}(t)=\sum_{i=1}^{N_{1}} a_{i} y\left(t-\tau_{i}\right)+\sum_{k=1}^{N_{2}}\left(b_{k}+\sum_{j=1}^{N_{3}} c_{j k} y\left(t-\tilde{\tau}_{j}\right)\right) u_{i}\left(t-h_{k}\right),
$$

where $N_{1}, N_{2}, N_{3}$ are nonnegative integers, $a_{i}, b_{i}, c_{i j} \in \mathbb{R}$ are constant parameters, and both state $\tau_{i}, \tilde{\tau}_{j}$ and input delays $h_{i}$ are considered in order to capture as much as possible the infinite dimensional dynamics of the system. However, this model is sufficiently simple and of small order to design some practically implementable control laws.

The identification has been done using a least-square method supported with global optimization algorithm NOMAD (Nonlinear Optimization by Mesh Adaptive Direct Search) (see, [38, 39, 40, 41]) required for optimal assignment of delays. The reader can refer to [24] for more details about identification of the considered bilinear model.

\subsection{Results of Identification}

The bilinear models have been identified for $N_{1}=N_{2}=2$ and $N_{3}=1$ or 2 . The precision of the models has been analyzed using the three indicators : $\epsilon$ is $L_{2}$-norm of the error, FIT index ${ }^{1}$ introduced in [42] and $\rho$ - the correlation between the experimental data and the identified model.

$\epsilon=\left\|y_{\text {exp }}-y_{\text {sim }}\right\|_{\mathbf{L}^{2}}, \quad \rho=\frac{\operatorname{cov}\left(y_{\text {exp }}, y_{\text {sim }}\right)}{\sigma_{y_{\text {exp }}} \sigma_{y_{s i m}}}, \quad \operatorname{FIT}=\left(1-\frac{\left\|y_{\text {exp }}-y_{\text {sim }}\right\|_{\mathbf{L}^{2}}}{\left\|y_{\text {exp }}-\bar{y}_{\text {exp }}\right\|_{\mathbf{L}^{2}}}\right) \times 100 \%$,

where $y_{\exp }$ is the output of the system obtained from the experiment, $y_{\text {sim }}$ is the output generated by the identified bilinear model $(3), \bar{y}_{\exp }$ is the mean value of $y^{\text {exp }}$, $\operatorname{cov}\left(y_{e x p}, y_{s i m}\right)$ is the covariance of $y_{\text {exp }}$ and $y_{s i m}$, but $\sigma_{y_{e x p}}$ and $\sigma_{y_{s i m}}$ are standard deviations of $y_{\text {exp }}$ and $y_{\text {sim }}$, respectively. The results are summarized in Tables 2 and 1 .

Table 1: Precision of the identified models

\begin{tabular}{c|c|c|c} 
& $\varepsilon$ & FIT & $\rho$ \\
\hline$N_{3}=1$ & 0.0495 & $87.56 \%$ & 0.9926 \\
\hline$N_{3}=2$ & 0.0341 & $91.44 \%$ & 0.9965
\end{tabular}

Its is worth stressing that the obtained models have very high precision comparing with the existing results [42]. The FIT index is improved for almost $30 \%$ using the model with only 8 parameters (see, Table I). The NARX (Nonlinear Autoregressive Exogenous Model) model obtained in [42] has hundreds of coefficients and FIT=59\%.

\footnotetext{
${ }^{1}$ FIT is the word "fit" in capital letters
} 
Table 2: Identified parameters of the models

\begin{tabular}{c|c|c} 
& $N_{3}=1$ & $N_{3}=2$ \\
\hline$\tau_{i}$ & {$[0.054 ; 0.006]$} & {$[0 ; 0.116]$} \\
\hline $\bar{\tau}_{j}$ & {$[0.054 ; 0.360]$} & {$[0.036 ; 0.001]$} \\
\hline$h_{k}$ & 0.054 & {$[0.045 ; 0.315]$} \\
\hline$a_{i}$ & {$[9.6468 ;-12.6195]$} & {$[4.4759 ; 0.3652]$} \\
\hline$b_{k}$ & 3.5632 & {$[29.1680 ;-28.7925 ;-24.1864 ; 23.4018]$}
\end{tabular}

\section{Setpoint Tracking Control Problem}

\subsection{Plant Model and Basic Assumptions}

For the design of a tracking control we use the simplest model obtained during the identification procedure, namely

$$
\begin{gathered}
\dot{y}(t)=a_{1} y(t-h)-a_{2} y(t-\tau)+(b-c y(t-h)+c y(t-\bar{\tau})) u(t-h), \\
y(s)=0, \quad u(s)=0 \quad \text { for } \quad s \leq 0,
\end{gathered}
$$

where $a_{1}>0, a_{2}>0, b>0, c>0, \bar{\tau}>0, h>0, \tau>0$ are constant parameters, $y(t) \in \mathbb{R}$ - output and $u \in \mathbf{L}_{\mathbb{R}}^{\infty}: u(t) \in\{0,1\}, t \geq 0$ is the input. Note that for any $u \in L_{\mathbb{R}_{+}}^{\infty}$ the considered system has a unique Caratheodory solution [43] at least locally.

We deal with a model of physical system. To exclude non-feasible dynamics we assume that the system (4), (5) has bounded positive solution for any input signal $u \in \boldsymbol{L}_{\mathbb{R}_{+}}^{\infty}: u(t) \in\{0,1\}$. The sufficient condition of positivity and boundedness of solutions to the system (4) is given by the next proposition proven in Appendix.

Proposition 1 If $c<a_{1},\left(a_{1}+c\right) \tau<a_{2} \tau<\frac{1}{e}$ and $\tau \leq h \leq \bar{\tau}$ then the system (4), (5) is positive and its solution is globally bounded for any input signal $u \in \boldsymbol{L}^{\infty}: u(t) \in$ $\{0,1\}$ as follows

$$
0 \leq y(t)<y_{\max }:=\frac{b}{a_{2}-a_{1}} \text { for all } t \geq 0
$$

\subsection{Sliding Mode Control for Time Delay Bilinear System}

The conventional sliding mode control methodology [25], [26], [27] is developed for delay-free systems. We emphasize again that, in our case, the choice of Sliding Mode control was motivated by the fact that the actuator is relay. In order to design the sliding mode control we need to compensate input delay using, for example, the prediction technique (see, e.g. [44], [45], [30], [31]). Developed originally for linear plants this idea can also be applied for bilinear systems under consideration.

A simple example of a predictor is given here :

Suppose that we study the positive system $\dot{x}(t)=a x(t)+b u(t-h)$ with $a<0, b>0$ and $u(t) \in\{0,1\}$. The maximum value of $x(t)$ is $x_{\max }=-\frac{b}{a}$, with $0<x(t)<x_{\max }$.

Consider the following prediction variable : $\sigma(t)=a x(t)+b \int_{t-h}^{t} u(s) d s$. Its derivative is: $\dot{\sigma}(t)=a x(t)+b u(t)$. Note that the control input $u$ is not delayed 
with respect to the sliding variable $\sigma$, so the conventional sliding mode design scheme can be utilized (see, [25]). Take $u(t)=\operatorname{sign}\left(\sigma(t)-\sigma^{*}\right)=\left\{\begin{array}{lll}1 & \text { if } & \sigma(t)-\sigma^{*}<0 \\ 0 & \text { if } & \sigma(t)-\sigma^{*}>0\end{array}\right.$, where $\sigma^{*}=a x^{*}+b$ and $x^{*} \in\left(0, x_{\max }\right)$ is the desired value to track. If $\sigma(t)>\sigma^{*}$ then $\dot{\sigma}(t)=a x(t)<0$ and, if $\sigma(t)<\sigma^{*}$ then $\dot{\sigma}(t)=a x(t)+b>a x_{\max }+b>0$.

Therefore, $\frac{d}{d t}\left(\sigma(t)-\sigma^{*}\right)\left(\sigma(t)-\sigma^{*}\right)<0$ and, according to [25], sliding mode arises on the surface $\sigma(t)-\sigma^{*}=0$ in a finite time, i.e. $\sigma(t)=\sigma^{*}$ for $t>t^{*}$.

Assuming $\bar{\tau}>h$ we introduce the following sliding variable for our model, according to $[31]$

$$
\sigma(t)=y(t)-a_{2} \int_{t-\tau}^{t} y(s) d s+c \int_{t-\bar{\tau}+h}^{t} y(s) d s+\int_{t-h}^{t} a_{1} y(s)+(b-c y(s)+c y(s-\bar{\tau}+h)) u(s) d s .
$$

Obviously, the variable $\sigma$ satisfies the equation

$$
\dot{\sigma}(t)=\left(a_{1}-a_{2}+c(1-u(t))\right) y(t)+c(u(t)-1) y(t-\bar{\tau}+h)+b u(t) .
$$

Proposition 2 If conditions of Proposition 1 hold and

$$
Q(j \omega) \neq 0 \quad \text { for } \quad \omega \neq 0,
$$

where $Q(s)=s+a_{2} e^{-s \tau}-\left(a_{2}-c\right) e^{-s h}-c e^{-s \bar{\tau}}, s \in \mathbb{C}$ and $j=\sqrt{-1}$, then the control law

$$
u(t)=\left\{\begin{array}{lll}
1 & \text { if } & \sigma(t)<\sigma^{*} \\
0 & \text { if } & \sigma(t)>\sigma^{*}
\end{array}\right.
$$

with $\sigma^{*}=y^{*}\left(1+a_{2}(h-\tau)+c(\bar{\tau}-h)\right)$ and $y^{*} \in\left(0, \frac{b}{a_{2}-a_{1}}\right)$ guarantees $y(t) \rightarrow y^{*}$ as $t \rightarrow+\infty$.

The proof of this proposition is given in Appendix, where it is shown that the control (8) guarantees finite-time convergence of the sliding variable $\sigma(t)$ to $\sigma^{*}$, so $\sigma(t)=\sigma^{*}$ for all $t \geq T$. It is worth stressing that when sliding mode arises the system motion is governed by the infinite dimensional dynamic system

$$
\sigma^{*}=y(t)+a_{2} \int_{t-h}^{t-\tau} y(s) d s+c \int_{t-\bar{\tau}}^{t-h} y(s) d s
$$

This means that our sliding surface $\sigma=\sigma^{*}$ is "inifine dimensional". Using condition (7) it is proven that the output $y(t)$ tends to $y^{*}$ asymptotically as $t \rightarrow \infty$.

Remark 1. Since

$$
\begin{gathered}
\operatorname{Re}(Q(j \omega))=a_{2} \cos (\tau \omega)-\left(a_{2}-c\right) \cos (h \omega)-c \cos (\bar{\tau} \omega) \\
\operatorname{Im}(Q(j \omega))=\omega-a_{2} \sin (\tau \omega)+\left(a_{2}-c\right) \sin (h \omega)+c \sin (\bar{\tau} \omega)
\end{gathered}
$$

then to check the condition (7) it is sufficient to consider $\omega \in\left(0,2\left(a_{2}+c\right)\right]$.

\subsection{Numerical Simulation of Setpoint Tracking Control}

Obviously, the plant model obtained by the identification (see, Table I, $N_{3}=1$ ) satisfies the proposition 1 with $a_{1}=9.6468, a_{2}=12.6195, c=2.6470, b=3.5632$, $\tau=0.006, h=0.054, \bar{\tau}=0.360$ and with the admissible setpoint value $y^{*} \in$ $\left(0, y_{\max }\right), y_{\max }=\frac{b}{a_{2}-a_{1}}=1.20$. According to Remark 1 the condition $(7)$ has been 


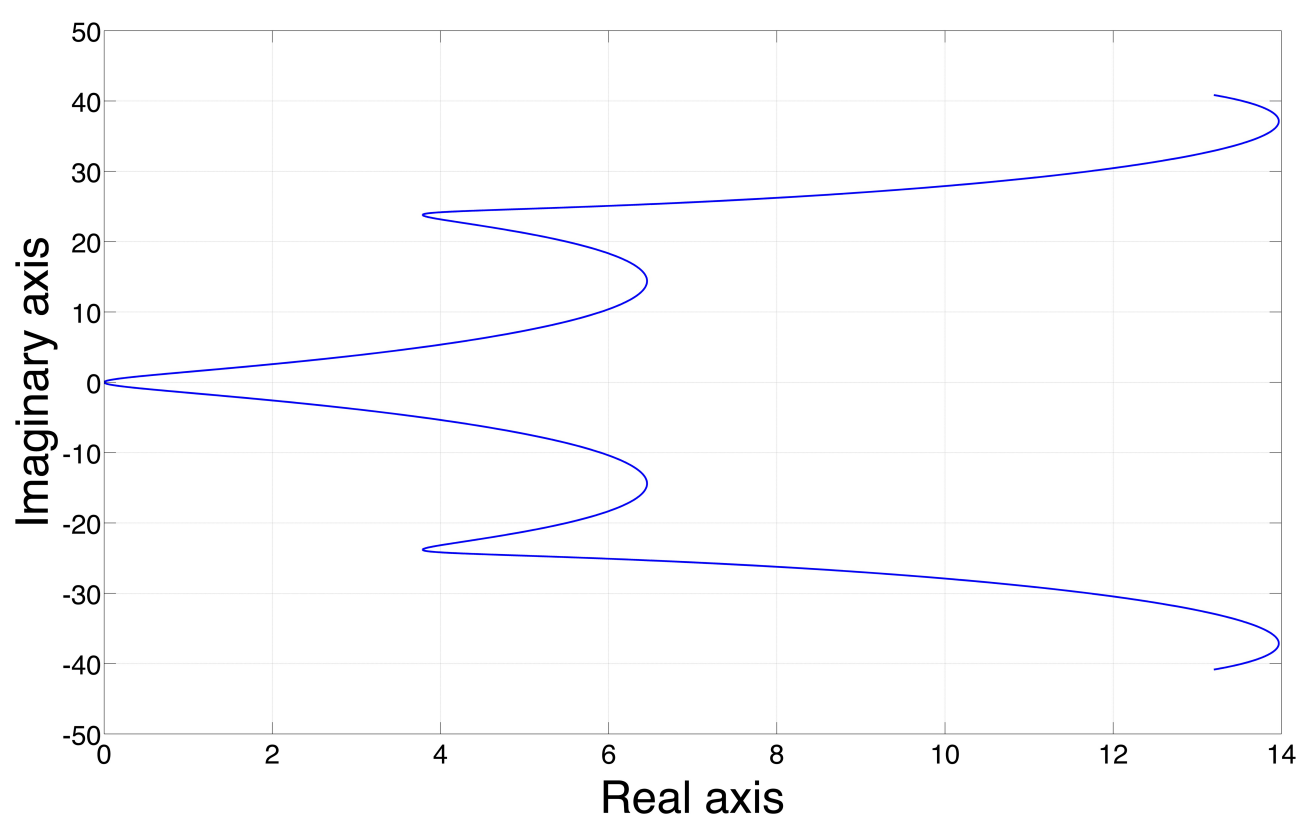

Figure 5: Parametric plot for $Q(j \omega)$ for $-2\left(a_{2}+c\right)<\omega<+2\left(a_{2}+c\right)$

validated graphically using the parametric plot of the function $Q$ in the complex plane (see, Fig. 5).

Results from a numerical simulation are depicted in Fig. 6 and Fig. 7 for $y^{*}=0.65$ $\left(\sigma^{*}=1.5702\right.$, respectively). The simulation has been done using explicit Euler method and a rather large step size $10^{-2}$ (corresponding to the technological limitations of most of the actuators). Such discretization step is selected in order to evaluate on simulations a possible amplitude of chattering [25], which always accompanies the sliding mode control. In addition, such step size corresponds to physical restrictions on the maximum frequency of many pneumatic actuators.

Fig. 7 shows that after approximately $0.65 \mathrm{~s}$, the sliding mode appears making the input oscillate between 0 and 1 at high frequency. Next, the output $y(t)$ converges to the desired setpoint $y^{*}$ with an error of the order $10^{-2}$. The numerical simulations have been also made for the smaller step size $10^{-4}$. They confirmed convergence of $y(t)$ to $y^{*}$ with an error of the order $10^{-4}$, which corresponds to numerical precision of the Euler method.

Fig. 8 plots the value of the sliding variable with respect to time and, except for the chattering phenomenon, it shows finite-time convergence to the sliding surface $\sigma(t)=\sigma^{*}$. For small time steps, $\sigma$ reaches $\sigma^{*}$ in finite-time and the reaching time is approximately $T_{\text {reach }}=0.65 \mathrm{~s}$.

Please note that all simulations are done using a zero initial condition for $y$.

\section{Optimal Control Problem}

\subsection{Model Description and Basic Assumptions}

Let us consider the functional differential equation

$$
\dot{y}(t)=A\left(y_{\tau}(t)\right)+\sum_{i=1}^{N_{3}}\left(b_{i}+B_{i}\left(y_{\tau}\left(t-h_{i}\right)\right)\right) u\left(t-h_{i}\right),
$$




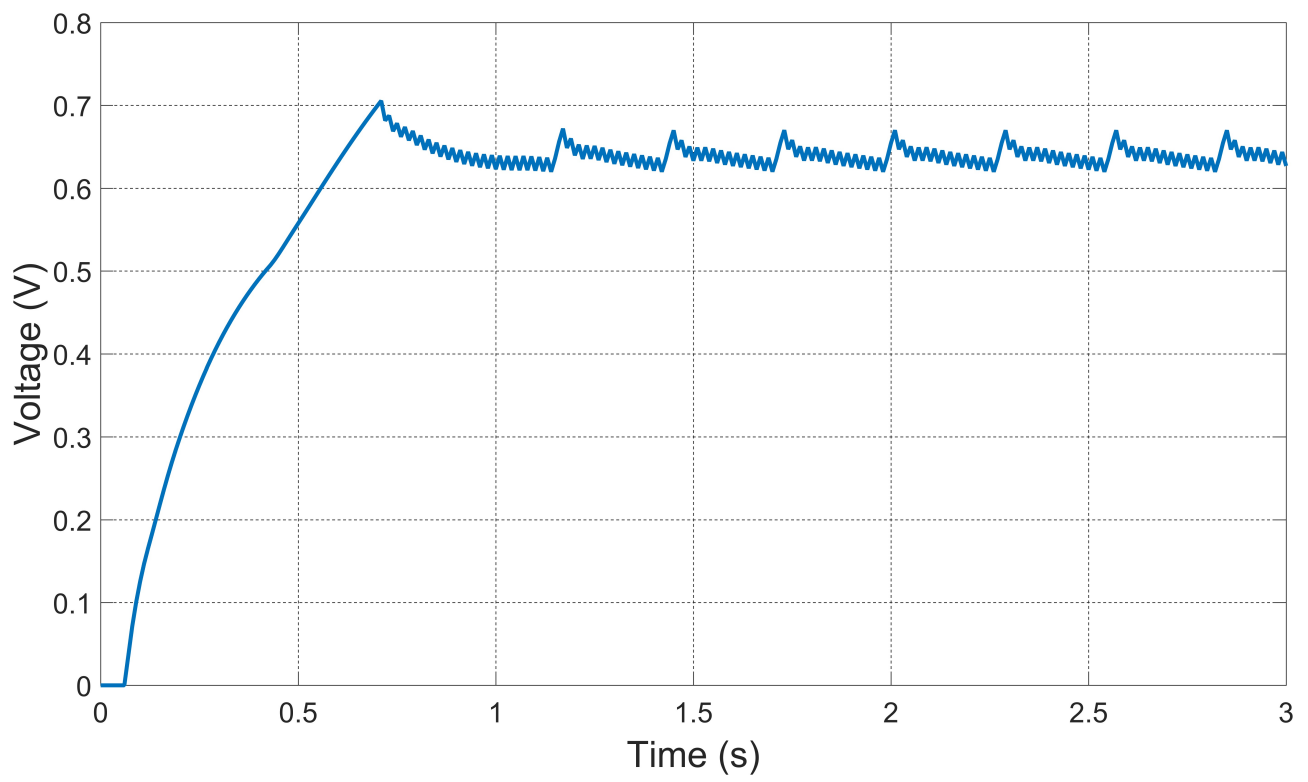

Figure 6: Application of the setpoint tracking control: Output of the system

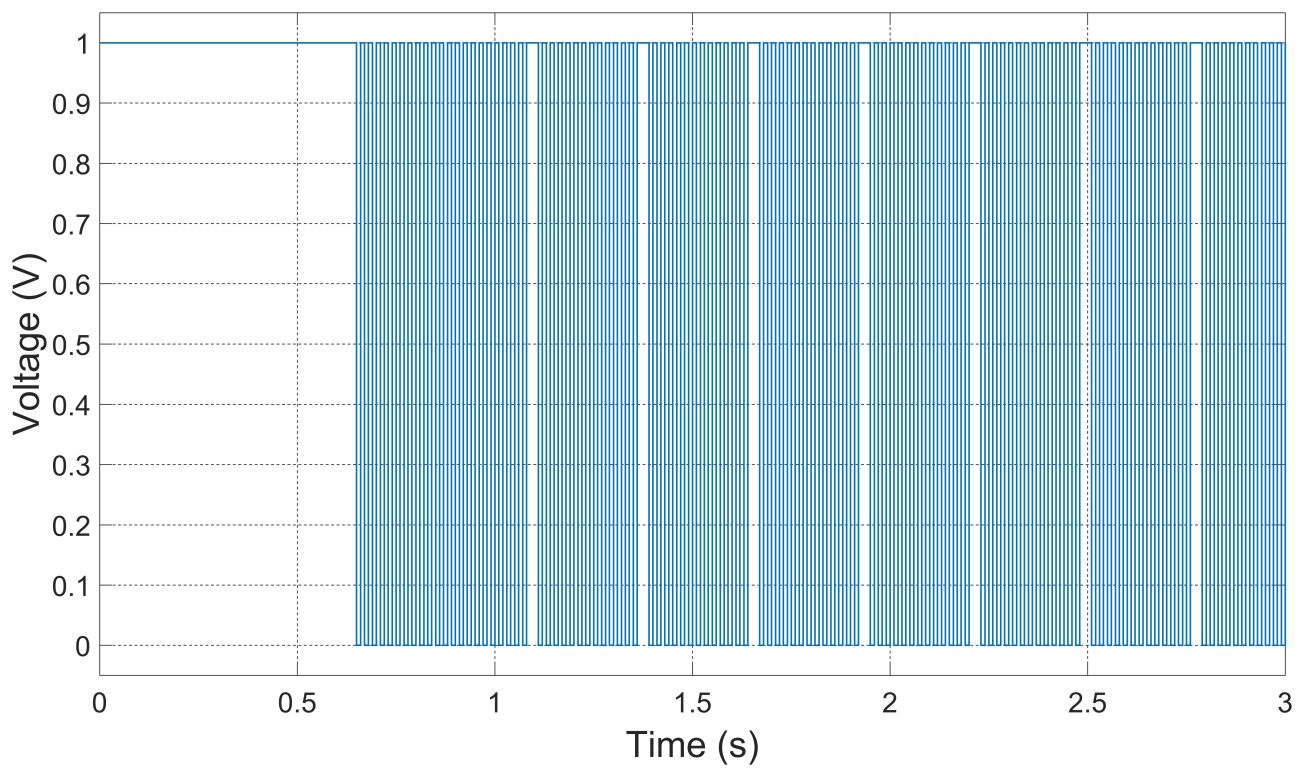

Figure 7: Application of the setpoint tracking control: Control signal

where $y_{\tau}(t) \in \mathbf{C}_{[-\tau, 0]}^{1}$ is the state of the system, $\left(y_{\tau}(t)\right)(s)=y(t+s)$ for $s \in[-\tau, 0]$, $A: \mathbf{C}_{[-\tau, 0]}^{1} \subset \mathbf{L}_{[-\tau, 0]}^{2} \rightarrow \mathbb{R}$ and $B_{i}: \mathbf{C}_{[-\tau, 0]}^{1} \subset \mathbf{L}_{[-\tau, 0]}^{2} \rightarrow \mathbb{R}$ are linear continuous functionals, $b_{i} \in \mathbb{R}_{+}$are positive constants, $u(t) \in\{0,1\}$ is the relay control input, $h_{i} \in \mathbb{R}_{+}$are input delays. For any $u \in \mathbf{L}_{\mathbb{R}_{+}}^{\infty}$ the considered system has a unique Caratheodory solution [43]. Similarly to the previous section we assume that the system (9) with $y(s)=0$ for all $s \leq 0$ has bounded positive solution for any input signal $u \in \boldsymbol{L}_{\mathbb{R}_{+}}^{\infty}: u(t) \in\{0,1\}$. We also assume that the class of admissible control inputs is restricted to $\omega$-periodic functions $u(t)=u(t+\omega), \forall t>0$. 


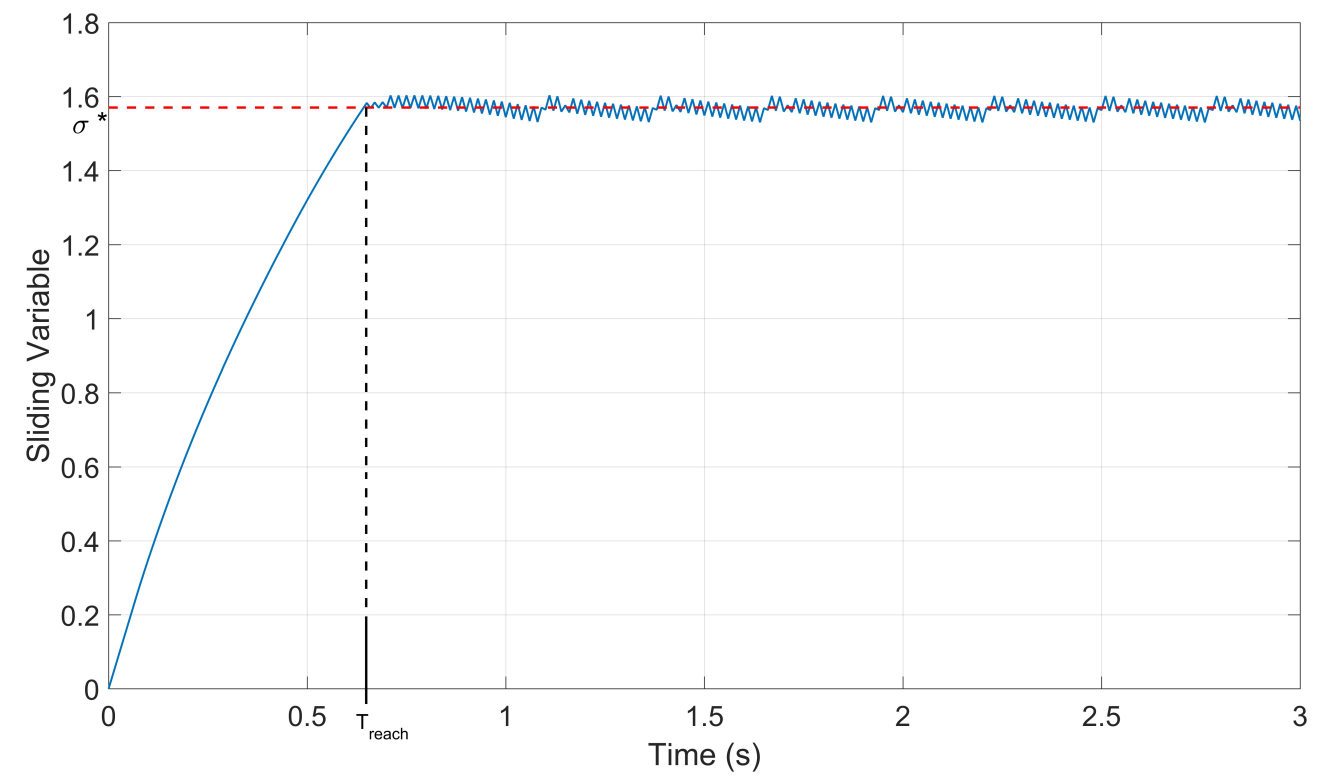

Figure 8: Application of the setpoint tracking control: Sliding variable

\subsection{Periodic Feedforward Control}

In the periodic case, the optimization problem $J(y, u) \rightarrow$ max subject to (9) considered over infinite interval of time can be reduced to the optimal control over finite time interval. Indeed, if for any $\omega$-periodic input $u \in \mathbf{L}_{\mathbb{R}_{+}}^{\infty}$ the system (9) has a unique stable $\omega$-periodic solution $y^{\omega}$ then

$$
J\left(y^{\omega}, u\right)=\frac{1}{\omega} \int_{0}^{\omega} \alpha y^{\omega}(s)-(1-\alpha) u(s) d s .
$$

To solve this optimization problem we need a proper algorithm of finding of periodic solutions to the system (9) with a given periodic control input $u$. Existence of periodic solution to a particular system (9) as well as algorithm for its finding is provided by the next theorem.

Theorem 1 ([46]) If $0=h_{0}<h_{1}<\ldots<h_{m}$ and

- a function $f: \mathbb{R}_{+} \times \mathbb{R}^{n(m+1)} \rightarrow \mathbb{R}$ is measurable and $\omega$-periodic: $f(t, \boldsymbol{x})=$ $f(t+\omega, \boldsymbol{x}), t \in \mathbb{R}_{+}, \boldsymbol{x} \in \mathbb{R}^{n(m+1)}$, and satisfies Lipschitz condition:

$$
|f(t, \boldsymbol{x})-f(t, \boldsymbol{y})| \leq \sum_{i=0}^{n-1} \sum_{j=0}^{m} l_{i j}\left|x_{i j}-y_{i j}\right|, \boldsymbol{x}, \boldsymbol{y} \in \mathbb{R}^{n(m+1)},
$$

where $l_{i j} \geq 0$ are constants, $\boldsymbol{x}=\left(x_{00}, x_{01}, \ldots, x_{i j}, \ldots\right) \in \mathbb{R}^{n(m+1)}$ and $\boldsymbol{y}=$ $\left(y_{00}, y_{01}, \ldots, y_{i j}, \ldots\right) \in \mathbb{R}^{n(m+1)}$,

- a liner functional $\mathcal{A}: \boldsymbol{C}_{\left[-h_{m}, 0\right]}^{n} \rightarrow \mathbb{R}$ is defined as

$$
\begin{gathered}
\mathcal{A} x_{h_{m}}(t)=\sum_{i=0}^{m} \sum_{j=0}^{n} a_{i j} x^{(j)}\left(t-h_{i}\right), \quad a_{i j} \in \mathbb{R}, \\
x_{h_{m}}(t)=x(t+s) \text { for } s \in\left[-h_{m}, 0\right],
\end{gathered}
$$

- the frequency $\theta=\frac{2 \pi}{\omega}$ satisfies the non-resonance conditions: $L(\mathrm{i} k \theta) \neq 0$ for $k=$ $0, \pm 1, \pm 2, \ldots$ where $p=0,1, \ldots, n-1$ and $L(\lambda)=\sum_{i=0}^{m} \sum_{j=0}^{n} a_{i j} \lambda^{j} e^{-h_{i} \lambda}$ is the characteristic quasi-polynomial of the operator $\mathcal{A}$, 
- the inequality $q=\sum_{p=0}^{n-1} l_{p} \sigma_{p}<1$ holds for $l_{p}=l_{p 0}+l_{p 1}+\ldots+l_{p m}$ and $\sigma_{p}=\max _{r \in \mathbb{R}}\left|\frac{(i r \theta)^{p}}{L(\mathrm{i} r \theta)}\right|$

then the equation $\mathcal{A} x_{h_{m}}(t)=f\left(t, x(t), x\left(t-h_{1}\right), \ldots, x^{(n-1)}\left(t-h_{m}\right)\right)$ has a unique $\omega$ periodic solution $x_{\omega} \in \boldsymbol{C}_{[0, \omega]}$,

which satisfies the estimate $\left\|x_{\omega}^{(i)}\right\|_{L_{[0, \omega]}^{2}} \leq \frac{\sigma_{i}}{1-q}\|f(t, \boldsymbol{O})\|_{L_{[0, \omega]}^{2}}, i=0,1, \ldots, n-1$ and can be found by means of iterations

$$
\mathcal{A} x_{h_{m}}^{[k+1]}(t)=f\left(t, x^{[k]}(t)\right), k=0,1,2, \ldots
$$

where $x^{[0]}$ is an arbitrary $\omega$-periodic function and $\boldsymbol{x}^{[k]}(t)=\left(x^{[k]}(t), x^{[k]}\left(t-h_{1}\right), \ldots, x^{[k]}(t-\right.$ $\left.\left.h_{m}\right), \ldots\right) \in \mathbb{R}^{n(m+1)}$ and the following estimate

$$
\left\|\frac{d^{i} x^{[k]}}{d t^{i}}-\frac{d^{i} x_{\omega}}{d t^{i}}\right\|_{L_{[0, \omega]}^{2}} \leq \frac{q^{k}}{1-q} \sigma_{i} \sum_{p=0}^{n-1} l_{p}\left\|\frac{d^{p} x^{[0]}}{d t^{p}}-\frac{d^{p} x_{\omega}}{d t^{p}}\right\|_{L_{[0, \omega]}^{2}}
$$

holds for $i=0,1,2, \ldots, n-1$.

To the best of our knowledge, the proof of Theorem 1 for $\mathbf{L}^{2}$ spaces has never been presented in English literature. Its proof given originally in [46] is sketched in Appendix.

The formula (11) provides simple recursive procedure for numerical finding of periodic solution with precision controlled by the formula (12). Combination of this algorithm with some infinite dimensional optimization procedure [47] allows us to find numerically an optimal input signal $u$ for a fixed period $\omega$. The corresponding algorithms are usually computationally hard. That is why, for practice, it is also important to provide a simple suboptimal algorithm. One has the following proposition, which is proved in Appendix.

Proposition 3 If for any $\omega$-periodic input signal $u \in \boldsymbol{L}_{\mathbb{R}}^{\infty}$ the poitive system (9) has a unique globally asymptotically stable periodic solution and $A(\mathbb{1})+\omega^{-1} \int_{0}^{\omega} \sum_{i=1}^{N_{2}} B_{i}\left(\bar{u}_{\tau}(s)\right) d s<$ 0 , then,

$$
J(y, u) \geq \tilde{J}(u)=\frac{1}{\omega}\left(\alpha-1-\frac{\alpha \sum_{i=1}^{N_{2}} b_{i}}{A(\mathbb{1})+\omega^{-1} \int_{0}^{\omega} \sum_{i=1}^{N_{2}} B_{i}\left(\bar{u}_{\tau}(s)\right) d s}\right) \int_{0}^{\omega} u(s) d s
$$

Therefore, if conditions of Proposition 3 holds then the sub-optimal control can be found by means of maximization of the functional $\tilde{J}(u)$.

If periodic control inputs are restricted to

$$
u_{\omega, t^{\prime}}(t)=\left\{\begin{array}{lll}
1 & \text { for } \quad t \in\left[k \omega, k \omega+t^{\prime}\right) \\
0 & \text { for } \quad t \in\left[k \omega+t^{\prime},(k+1) \omega\right),
\end{array}\right.
$$

then, in the view of Proposition 3, a quasi optimal solution to (2) can be found from the finite dimensional optimization problem: $\tilde{J}\left(u_{\omega, t^{\prime}}\right) \rightarrow$ max. Such class of input signals is motivated by natural practical demands to minimize the number of switchings. 
In this case, the condition $A(\mathbb{1})+\omega^{-1} \int_{0}^{\omega} \sum_{i=1}^{N_{2}} B_{i}\left(\bar{u}_{\tau}(s)\right) d s<0$ of 3 simplifies to $A(\mathbb{1})+\frac{t^{\prime}}{\omega} \sum_{i=1}^{N_{2}} B_{i}(\mathbb{1})<0$, and $\tilde{J}(u)$ to :

$$
\tilde{J}(u)=\left(\alpha-1-\frac{\alpha \sum_{i=1}^{N_{2}} b_{i}}{A(\mathbb{1})+\frac{t^{\prime}}{\omega} \sum_{i=1}^{N_{2}} B_{i}(\mathbb{1})}\right) \frac{t^{\prime}}{\omega}
$$

This optimization problem can be solved analytically for $\tilde{J}$. For any fixed value $\omega_{0}$ of $\omega$, the value of $t^{\prime}$ noted by $t_{0}^{\prime}$ is given by :

$$
t_{0}^{\prime}= \begin{cases}\omega & \text { if } \alpha=0 \\ \omega \operatorname{sat}_{[0,1]}\left(\frac{-A(\mathbb{1})}{\sum_{i=1}^{N_{2}} B_{i}(\mathbb{1})}-\frac{\sqrt{\frac{\alpha}{(\alpha-1)} A(\mathbb{1})\left(\sum_{i=1}^{N_{2}} b_{i}\right)}}{\sum_{i=1}^{N_{2}} B_{i}(\mathbb{1})}\right) & \text { if } \alpha=1 \\ \text { if } 0<\alpha<1\end{cases}
$$

where sat $_{[0,1]}$ is the saturation function on the interval $[0,1] \operatorname{such}$ that $\operatorname{sat}_{[0,1]}(x)=$ $x$ for $0 \leq x \leq 1, \operatorname{sat}_{[0,1]}(x)=1$ for $x>1$ and $\operatorname{sat}_{[0,1]}(x)=0$ for $x<0$

It is worth stressing that the identified model of the flow control system (see, Table I) satisfies Proposition 3.

\subsection{Numerical simulation results}

Let us find the suboptimal feedforward control of the form (13) for our system with two input delays as presented in Section 5.2. It is easy to compute that $A(\mathbb{1})=$ $-3.7488<0$ and $A(\mathbb{1})+B(\mathbb{1})=-4.1578<0$, then the conditions of Proposition 3 are fulfilled for every couple $\left(\omega, t^{\prime}\right)$.

A numerical simulation of the model (9) with the coefficients chosen in the column $N_{3}=2$ of Table I can be found in Fig. 9. This simulation was done for $\alpha=0.46$ and $\omega=0.25$, leading to $t^{\prime}=0.1119$ and the duty cycle $\frac{t^{\prime}}{\omega} \times 100 \%=44.76 \%$. The value of the cost function for this simulation is $J=-0.0339$. For comparison, and for identical values of $\alpha$ and $\omega$, taking a constant control equal to one give $J=-0.0711$ and a control with a duty cycle of $50 \%$ gives $J=-0.0389$. Another simulation with $\alpha=0.48$ can be found in Fig. 10. It is to be noted that, in the case of our model, the choice of alpha requires great precision as the saturation of $t_{s w}$ occurs for $\alpha<0.436$ and $\alpha>0.488$. Therefore, the choice of the parameter $\alpha$ greatly depends on the system studied and its range and effect may vary from one experiment to another.

Please note that all simulations are done using a zero initial condition for $y$.

\section{Discussion and Conclusions}

In the paper the problem of model-based control of separated flows is studied.

The bilinear time delay model of turbulent flow introduced in [24] is considered and identified. It is shown that both sub-optimal (feedforward) and robust(feedback) control laws can be designed based on the identified model. Classical control approaches like sliding mode design can be utilized for this purpose. The experimental tests of the proposed control strategies as well as their extensions to more exact models of separated flows are planned for the future work. 


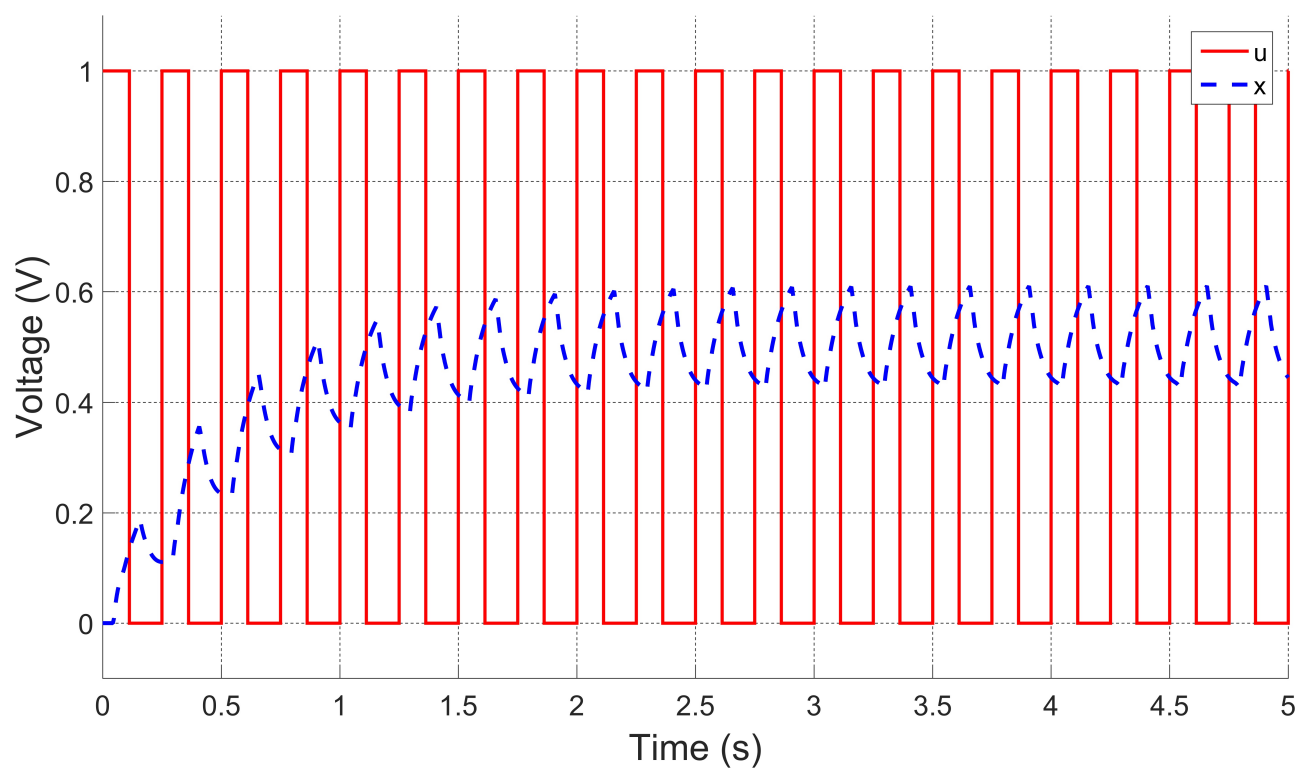

Figure 9: Application of the feedforward control with $\alpha=0.46$

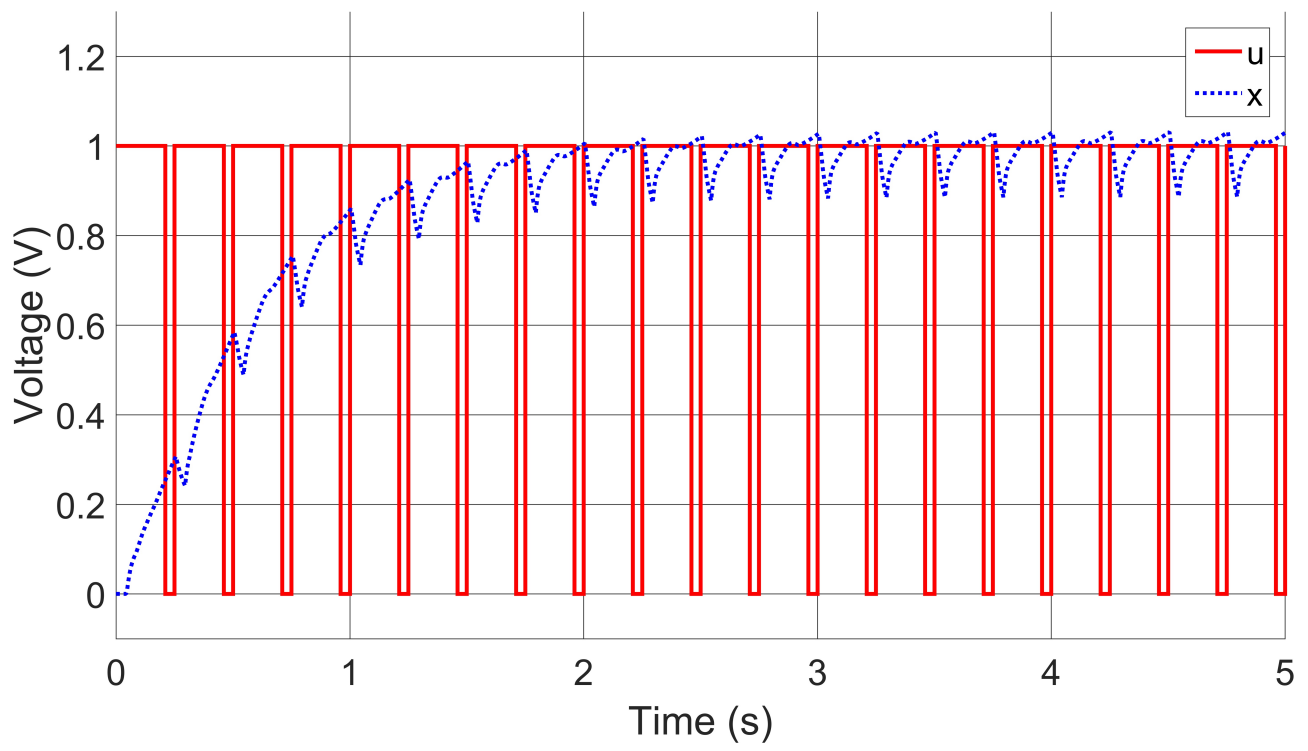

Figure 10: Application of the feedforward control with $\alpha=0.48$

Chattering reduction of Sliding Mode control was not studied in this paper. Today the way for chattering reduction is to use Higher Order Sliding Mode. However, till now there is no efficient scheme for its application to time-delay bilinear systems. We consider this problem as important direction for future research. Another possible direction is related to disturbance reduction and noise sensitivity analysis using for example, the results of the papers [31], [33], [48]. This would also allow us to cover a wider range of flow types and experiments. 


\section{Appendix}

\subsection{Positive systems with time-delay}

Lemma 1 If $a>0, \tau>0$ and $a \tau<\frac{1}{e}$ then the system $\dot{z}(t)=-a z(t-\tau)+f(t), z(s)=$ 0 for $s \leq 0$ is positive for any non-negative $f \in \boldsymbol{L}_{\mathbb{R}}^{\infty}$, i.e. $z(t) \geq 0$ if $f(t) \geq 0$ for all $t \geq 0$.

Proof. See Lemma 4 of [49] or Corollary 15.9 from [50].

Lemma 2 Let the system $\dot{z}(t)=-a z(t-\tau)+b$ with $z(s)=0$ for $s \leq 0$ be positive and $a>0, b>0,0 \leq a \tau<\ln (2)$. Then it has a unique solution defined on $\mathbb{R}_{+}$such that $0<z(t)<\frac{b}{a}$ and $\dot{z}(t)>0$ for all $t>0$.

Proof. For existence and uniqueness of solution of time-delay systems see [35]. Let us suppose a contrary, i.e. there exists $t^{*}>0$ such that $z\left(t^{*}\right)=\frac{b}{a}$, but $z(t)<\frac{b}{a}$ for all $t>t^{*}$. This immediately implies that $\dot{z}(t)>0$ and $z(t)>0$ for all $t \in\left(0, t^{*}\right]$.

Let us denote $p(t)=z(t)-a \int_{t-\tau}^{t} z(s) d s$. Hence, we have

$$
\dot{p}(t)=-a z(t)+b=-a p(t)+b-a^{2} \int_{t-\tau}^{t} z(s) d s
$$

and

$$
\begin{aligned}
z(t) & =\frac{b\left(1-e^{-a t}\right)}{a}+a \int_{t-\tau}^{t} z(s) d s-a^{2} \int_{0}^{t} e^{-a(t-\sigma)} \int_{\sigma-\tau}^{\sigma} z(s) d s d \sigma \\
& =\frac{b\left(1-e^{-a t}\right)}{a}+a \int_{t-\tau}^{t} z(s) d s-a \int_{0}^{t} e^{-a(t-\sigma)} \int_{t-\tau}^{t} z(\sigma-t+s) d s d \sigma \\
& =\frac{b\left(1-e^{-a t}\right)}{a}+a \int_{t-\tau}^{t}\left(z(s)-a \int_{0}^{t} e^{-a(t-\sigma)} z(\sigma-t+s) d \sigma\right) d s \\
& =\frac{b\left(1-e^{-a t}\right)}{a}+a \int_{t-\tau}^{t}\left(z(s)-a \int_{-t}^{0} e^{a \sigma} z(s+\sigma) d \sigma\right) d s \\
& =\frac{b\left(1-e^{-a t}\right)}{a}+a \int_{t-\tau}^{t}\left(z(s)-a e^{-a s} \int_{s-t}^{s \sigma} z(\sigma) d \sigma\right) d s \\
& =\frac{b}{a}\left(1-e^{-a t}\right)+a \int_{t-\tau}^{t} e^{-a s} f(s, t) d s,{ }^{a}{ }^{a}(z)
\end{aligned}
$$

where $f(s, t)=e^{a s} z(s)-a \int_{s-t}^{s} e^{a \sigma} z(\sigma) d \sigma$. Since for $s \in[t-\tau, t]$ and $0<t \leq t^{*}$ we have

$$
\frac{\partial f}{\partial s}=e^{a s} \dot{z}(s)+a e^{a s} z(s)-a e^{a s} z(s)+a e^{a(s-t)} z(s-t) \geq 0
$$

then $z(t) \leq \frac{b\left(1-e^{-a t}\right)}{a}+a f(t, t) \int_{t-\tau}^{t} \frac{d s}{e^{a s}}=\frac{b\left(1-e^{-a t}\right)}{a}+f(t, t) \frac{e^{a \tau}-1}{e^{a t}}$ or, equivalently, $z(t) \leq \frac{b\left(1-e^{-a t}\right)}{a\left(2-e^{a \tau}\right)}-\frac{a\left(e^{a \tau}-1\right)}{2-e^{a \tau}} \int_{0}^{t} e^{-a(t-\sigma)} z(\sigma) d \sigma$. Hence, $z(t) \leq w(t)$, where $w(t)$ satisfies the integral equation $w(t)=\frac{b\left(1-e^{-a t}\right)}{a\left(2-e^{a \tau}\right)}-\frac{a\left(e^{a \tau}-1\right)}{2-e^{a \tau}} \int_{0}^{t} e^{-a(t-\sigma)} w(\sigma) d \sigma, w(0)=0$ 
or, equivalently,

$$
\dot{w}(t)=-a\left(1+\frac{e^{a \tau}-1}{2-e^{a \tau}}\right) w(t)+\frac{b}{2-e^{a \tau}}=\frac{-a w(t)+b}{2-e^{a \tau}} .
$$

Therefore, we derive that $z(t) \leq \frac{b}{a}\left(1-e^{-\frac{a}{2-e^{a \tau} t}}\right)<\frac{b}{a}$ for all $t>0$. This contradicts our supposition.

\subsection{Proof of Proposition 1}

I. Let us consider the system $\dot{y}(t)=-a_{2} y(t-\tau)+f(t), y(s)=0, s \leq 0$, where $f$ is a locally integrable function. If $f(t) \geq 0$ and $a_{2} \tau \leq e^{-1}$ then the delay-dependent positivity conditions hold (see, Lemma 1 ) and $y(t) \geq 0$, for all $t \geq 0$. On the one hand, if $a_{1} \geq c$ and $y(s) \geq 0$ for $s \leq t$ then $f(t)=a_{1} y(t-h)+(b-c y(t-h)+c y(t-$ $\bar{\tau})) u(t-h) \geq 0$. Therefore, using the method of steps (i.e. considering sequentially the intervals $[0, h],[h, 2 h] \ldots)$ we prove positivity of the system (4), (5).

II. Now let us prove boundedness of solutions. Suppose the contrary: there exists an input signal $u(t)$ and an instant of time $t^{*}>0: y\left(t^{*}\right)=y_{\max }$ and $y(s)<y_{\max }$ for $s<t^{*}$.

In this case, since $b-c y(t-h)+c y(t-\bar{\tau}) \geq b-c y(t-h)=\left(a_{2}-a_{1}\right) y_{\max }-c y(t-h)>0$ for all $t \in\left[0, t^{*}\right]$ then $y(s) \leq y_{\mathbb{1}}(s)$ for all $s \leq t^{*}$, where $y_{\mathbb{1}}$ is the solution to the positive system $\dot{y}_{\mathbb{1}}(t)=\left(a_{1}-c\right) y_{\mathbb{1}}(t-h)-a_{2} y_{\mathbb{1}}(t-\tau)+c y_{\mathbb{1}}(t-\bar{\tau})+b$. Let us prove boundedness of solutions of the latter system for this purpose let us study the auxiliary system

$$
\dot{z}(t)=-\left(a_{2}-a_{1}\right) z(t-\tau)+b, \quad z(s)=0 \text { for } s \leq 0 .
$$

For $\Delta(t)=z(t)-y_{\mathbb{1}}(t)$ we derive $\dot{\Delta}(t)=-a_{2} \Delta(t-\tau)+a_{1} z(t-\tau)-\left(a_{1}-c\right) y_{\mathbb{1}}(t-h)-$ $c y_{1}(t-\bar{\tau})$. According to Lemma 2 the function $z$ satisfies the inequalities $0<z(t)<$ $b /\left(a_{2}-a_{1}\right)=y_{\max }$ and $\dot{z}(t)>0$ for all $t>0$. Hence, $z(t-\tau) \geq z(t-h) \geq z(t-\bar{\tau})$ and

$$
\dot{\Delta}(t)=-a_{2} \Delta(t-\tau)+\left(a_{1}-c\right) \Delta(t-h)+c \Delta(t-\bar{\tau})+\eta(t),
$$

where $\eta(t)=a_{1} z(t-\tau)-\left(a_{1}-c\right) z(t-h)-c z(t-\bar{\tau}) \geq 0$ and $\Delta(s)=0$ for $s \leq 0$. Since the latter system is positive (see, the first part of this proof) then $\Delta(t) \geq 0$ and $y_{\mathbb{1}}(t) \leq z(t)<y_{\max }$ for all $t \geq 0$. This contradicts our supposition.

\subsection{Proof of Proposition 2}

Firs of all, let us note that $0<y(t)<\frac{b}{a_{2}-a_{1}}$ for all $t>0$ due to Proposition 1 .

I. Since the system (4) is positive, then $y(t) \geq 0$ for all $t \geq 0$. Moreover, if $u(t)=0$ and $y(t)>0$ then $\dot{\sigma}(t)<0$, but if $u(t)=1$ and $y(t)<\frac{b}{a_{2}-a_{1}}$ then $\dot{\sigma}(t)>0$. Therefore, while $0<y(t)<\frac{b}{a_{2}-a_{2}}$ we have $\left(\sigma(t)-\sigma^{*}\right) \frac{d}{d t}\left(\sigma(t)-\sigma^{*}\right)<0$. Obviously, $\sigma(0)=0$. To guarantee existence of sliding mode we just need to show that the state $\sigma(t)=\sigma^{*}>0$ is reachable in a finite time $t=t^{*}>0$. Let us suppose contrary: $\sigma(t)<\sigma^{*}$ for all $t>0$. This means that $u(t)=1$ for all $t>0$ and

$$
\dot{y}(t)=\left(a_{1}-c\right) y(t-h)-a_{2} y(t-\tau)+c y(t-\bar{\tau})+b .
$$

Using the last identity let us rewrite the formula (6) as

$$
\sigma(t)=y(t)-a_{2} \int_{t-\tau}^{t} y(s) d s+c \int_{t-\bar{\tau}+h}^{t} y(s) d s+\int_{t-h}^{t} \dot{y}(s+h)+a_{2} y(s+h-\tau) d s=
$$




$$
2 y(t)-y(t-h)+a_{2} \int_{t}^{t+h-\tau} y(s) d s+c \int_{t-\bar{\tau}+h}^{t} y(s) d s .
$$

Hence, $\sigma(t) \geq \sigma^{*}$ if $y(t) \geq y^{*}$. Let us show that there exists $t^{*}>0$ such that $y(t)>y^{*}$ for all $t>t^{*}$. Since $\dot{y}(t)>-a_{2} y(t-\tau)+b$ for all $t>0$ then, obviously, there exists $t_{1}>0$ such that $y(t)>\frac{b}{a_{2}}$ for all $t>t_{1}$. In this case, we derive $\dot{y}(t)>-a_{2} y(t-\tau)+b\left(1+\frac{a_{1}}{a_{2}}\right)$ for all $t>t_{1}+\bar{\tau}$ and there exists $t_{2}>t_{1}+\bar{\tau}$ such that $y(t)>\frac{b}{a_{2}}\left(1+\frac{a_{1}}{a_{2}}\right)$ for all $t>t_{2}$, and so on. Therefore, for $t>t_{i}$ we derive

$$
y(t)>\frac{b}{a_{2}}\left(1+\frac{a_{1}}{a_{2}}+\ldots+\left(\frac{a_{1}}{a_{2}}\right)^{i-1}\right)=\frac{b\left(1-\left(\frac{a_{1}}{a_{2}}\right)^{i}\right)}{a_{2}-a_{1}}
$$

and for some $i^{*}$ we have $y\left(t_{i^{*}}\right)>y^{*} \in\left(0, \frac{b}{a_{2}-a_{1}}\right)$. Therefore, the sliding mode existence condition [25] holds and $\sigma(t)=\sigma^{*}, \forall t>t^{*}$.

II. Using the equivalent control method [25] we derive $u_{e q}(t)=\frac{\left(a_{2}-a_{1}-c\right) y(t)+c y(t-\bar{\tau}+h)}{b-c y(t)+c y(t-\bar{\tau}+h)}$ and

$$
\begin{gathered}
\sigma^{*}=y(t)-a_{2} \int_{t-\tau}^{t} y(s) d s+c \int_{t-\bar{\tau}+h}^{t} y(s) d s+\int_{t-h}^{t} a_{2} y(s)-c y(s)+c y(s-\bar{\tau}+h) d s= \\
y(t)+a_{2} \int_{t-h}^{t-\tau} y(s) d s+c \int_{t-\bar{\tau}}^{t-h} y(s) d s
\end{gathered}
$$

for all $t>t^{*}$. Introducing the variable $\Delta(t)=y(t)-y^{*}$ we obtain the equation

$$
\Delta(t)+a_{2} \int_{t-h}^{t-\tau} \Delta(s) d s+c \int_{t-\bar{\tau}}^{t-h} \Delta(s) d s=0 .
$$

It has the characteristic equation $\frac{1}{s} Q(s)=0, s \in \mathbb{C}$. We have already proven that all solutions of the closed-loop system are bounded (see, Proposition 1) and the sliding mode exists for all $t>t^{*}$, so the equation (16) does not have unbounded dynamics. The condition $Q(j \omega) \neq 0$ for all $\omega \neq 0$ implies that this equation does not have nonconstant periodic solutions. So, the only stable solution is $\Delta(t) \equiv C$, where $C \in \mathbb{R}$ is some constant. Since $1+a_{2}(h-\tau)+c(\bar{\tau}-h)>0$ then from the equation for $\Delta(t)$ we immediately derive $C=0$ and $y(t) \rightarrow y^{*}$ as $t \rightarrow \infty$.

\subsection{Elements of Averaging Analysis}

Let us introduce some additional notations.

- $\mathbf{L}_{\Omega}^{2}$ is the Hilbert space of quadratically integrable functions $\Omega \subseteq \mathbb{R} \rightarrow \mathbb{R}$ with the norm $\|\cdot\|_{\mathbf{L}^{2}}$ induced by the inner product $\xi \cdot \eta=\int_{\Omega} \xi(s) \eta(s) d s$.

- If $\tau>0, \xi \in \mathbf{L}_{\mathbb{R}}^{\infty}$ and $t \in \mathbb{R}$ then

$$
\bar{\xi}_{\tau}(t) \in \mathbf{L}_{[-\tau, 0]}^{\infty}:\left(\bar{\xi}_{\tau}(t)\right)(\sigma)=\xi(t-\sigma) \text { for } \sigma \in[-\tau, 0],
$$


- For $\xi, \nu \in \mathbf{L}_{\Omega}^{\infty}$ with $\mathbb{R}_{+} \subset \Omega$ let us define the operation

$$
\langle\xi, \nu\rangle:=\lim _{T \rightarrow+\infty} \frac{1}{T} \int_{0}^{T} \xi(\tau) \nu(\tau) d \tau,
$$

which, in particular, defines the scalar product in the space $\mathbf{L}_{\mathbb{R}_{+}}^{\infty}$.

- For $\xi, \nu \in \mathbf{L}_{\mathbb{R}}^{\infty}$ let us define $\left\langle\xi_{\tau}, \nu\right\rangle \in \mathbf{L}_{[-\tau, 0]}^{\infty}$ as

$$
\left\langle\xi_{\tau}, \nu\right\rangle(\sigma):=\lim _{T \rightarrow+\infty} \frac{1}{T} \int_{0}^{T} \xi(s+\sigma) \nu(s) d s \text { for } \sigma \in[-\tau, 0] .
$$

Lemma 3 If $\xi, \nu \in \boldsymbol{L}_{\mathbb{R}}^{\infty}$ and $\tau \in \mathbb{R}_{+}$then $\left\langle\xi_{\tau}, \nu\right\rangle=\left\langle\xi, \bar{\nu}_{\tau}\right\rangle$.

Proof. The change of integration variable $\theta=s+\sigma$ gives

$$
\left\langle\xi_{\tau}, \nu\right\rangle(\sigma)=\lim _{T \rightarrow+\infty} \int_{0}^{T} \frac{\xi(s+\sigma) \nu(s)}{T} d s=\lim _{T \rightarrow+\infty} \int_{0}^{T+\sigma} \frac{\xi(\theta) \nu(\theta-\sigma)}{T} d \theta=\left\langle\xi, \bar{\nu}_{\tau}\right\rangle(\sigma)
$$

for $\sigma \in[-\tau, 0]$.

This proposition immediately implies the following

Corollary 1 If $\xi \in \boldsymbol{L}_{\mathbb{R}}^{\infty}$ and $\tau \in \mathbb{R}_{+}$then $\left\langle\xi_{\tau}, \mathbb{1}\right\rangle \in \boldsymbol{C}_{[-\tau, 0]}$ is the constant function: $\left\langle\xi_{\tau}, \mathbb{1}\right\rangle(\sigma)=\langle\xi, \mathbb{1}\rangle$ for all $\sigma \in[-\tau, 0]$, where $\mathbb{1} \in \boldsymbol{C}_{\mathbb{R}}$ is the unit constant function (i.e. $\mathbb{1}(s)=1$ for all $s \in \mathbb{R})$.

Lemma 4 If $\Phi: \boldsymbol{L}_{[-\tau, 0]}^{2} \rightarrow \mathbb{R}_{+}$is a linear continuous functional then $\left\langle\Phi\left(\xi_{\tau}\right), \nu\right\rangle=$ $\Phi\left(\left\langle\xi_{\tau}, \nu\right\rangle\right)$ for any $\xi, \nu \in \boldsymbol{L}_{\mathbb{R}}^{\infty}$.

Proof. Since $\Phi$ is the linear continuous functional $\mathbf{L}_{[-\tau, 0]}^{2} \rightarrow \mathbb{R}_{+}$then by Riesz Theorem [51] it can be presented as

$$
\Phi(\eta)=\mu \cdot \eta=\int_{-\tau}^{0} \mu(s) \eta(s) d s, \quad \eta \in \mathbf{L}_{[-\tau, 0]}^{2}
$$

with some $\mu \in \mathbf{L}_{[-\tau, 0]}^{2}$. Hence,

$$
\frac{1}{T} \int_{0}^{T} \Phi\left(\xi_{\tau}(s)\right) \nu(s) d s=\frac{1}{T} \int_{0}^{T} \int_{-\tau}^{0} \mu(\sigma) \xi(s+\sigma) d \sigma \nu(s) d s=\int_{-\tau}^{0} \mu(\sigma) \frac{1}{T} \int_{0}^{T} \xi(s+\sigma) \nu(s) d s d \sigma .
$$

Taking the limit for both sides we complete the proof.

Corollary 2 If $\Phi: \boldsymbol{L}_{[-\tau, 0]}^{2} \rightarrow \mathbb{R}_{+}$is a linear continuous functional then

$$
\lim _{T \rightarrow+\infty} \frac{1}{T} \int_{0}^{T} \Phi\left(\xi_{\tau}(s)\right) d s=\langle\xi, \mathbb{1}\rangle \Phi(\mathbb{1}),
$$

for any $\xi \in \boldsymbol{L}_{\mathbb{R}}^{\infty}$, where $\mathbb{1} \in \boldsymbol{C}_{\mathbb{R}_{+}}$is the unit constant function.

Lemma 5 If $A: C_{[-\tau, 0]}^{1} \subset \boldsymbol{L}_{[-\tau, 0]}^{2} \rightarrow \mathbb{R}$ and $B: C_{[-\tau, 0]}^{1} \subset \boldsymbol{L}_{[-\tau, 0]}^{2} \rightarrow \mathbb{R}$ are linear continuous functionals and the system (9) has bounded solution for any $u \in \boldsymbol{L}_{\mathbb{R}}^{\infty}$ then

$$
\sum_{i=1}^{N_{2}} b_{i}\langle u, \mathbb{1}\rangle+\left\langle y, B_{i}\left(\bar{u}_{\tau}\right)\right\rangle+\langle y, \mathbb{1}\rangle A(\mathbb{1})=0,
$$

where $\mathbb{1}$ is the unit constant function, $y$ is a solution to (9) with $u \in \boldsymbol{L}_{\mathbb{R}}^{\infty}$ and $\bar{u}_{\tau}(t) \in$ $\boldsymbol{L}_{[-\tau, 0]}^{\infty}:\left(\bar{u}_{\tau}(t)\right)(\sigma)=u(t-\sigma)$ for $\sigma \in[-\tau, 0]$. 
Proof. From the equation (9) we have

$$
\begin{aligned}
\int_{0}^{T} \frac{\dot{y}(s)}{T} d s & =\int_{0}^{T} \frac{A\left(y_{\tau}(s)\right)}{T}+\sum_{i=1}^{N_{2}} \frac{b_{i}+B_{i}\left(y_{\tau}\left(s-h_{i}\right)\right) u\left(s-h_{i}\right)}{T} d s \\
& =A\left(\int_{0}^{T} \frac{y_{\tau}(s)}{T} d s\right)+\sum_{i=1}^{N_{2}} \frac{1}{T} \int_{-h_{i}}^{T-h_{i}} b_{i} u(s)+B_{i}\left(y_{\tau}(s)\right) u(s) d s .
\end{aligned}
$$

Since the function $y$ is bounded then $\lim _{T \rightarrow+\infty} \frac{1}{T} \int_{0}^{T} \dot{y}(s) d s=\lim _{T \rightarrow+\infty} \frac{1}{T}(y(T)-$ $y(0))=0$ and using Corollary 1 and Lemma 4 we derive $\lim _{T \rightarrow+\infty} \frac{1}{T} \int_{0}^{T} A\left(y_{\tau}(s)\right) d s=$ $\langle y, \mathbb{1}\rangle A(\mathbb{1})$. Since

$$
\lim _{T \rightarrow+\infty} \frac{1}{T} \int_{-h_{i}}^{T-h_{i}} B_{i}\left(y_{\tau}(s)\right) u(s) d s=\left\langle B_{i}\left(y_{\tau}\right), u\right\rangle
$$

then

$$
0=\langle y, \mathbb{1}\rangle A(\mathbb{1})+\langle u, \mathbb{1}\rangle \sum_{i=1}^{N_{2}} b_{i}+\left\langle B_{i}\left(y_{\tau}\right), u\right\rangle .
$$

Finally, Lemmas 3 and 4 imply $\left\langle B_{i}\left(y_{\tau}\right), u\right\rangle=B_{i}\left(\left\langle y_{\tau}, u\right\rangle\right)=B_{i}\left(\left\langle y, \bar{u}_{\tau}\right\rangle\right)=\left\langle y, B_{i}\left(\bar{u}_{\tau}\right)\right\rangle$.

\subsection{Proof of Proposition 3}

In the periodic case, we derive $\langle u, \mathbb{1}\rangle=\frac{1}{\omega} \int_{0}^{\omega} u(s) d s,\langle y, \mathbb{1}\rangle=\frac{1}{\omega} \int_{0}^{\omega} y^{\omega}(s) d s$ and $\left\langle y, B_{i}\left(\bar{u}_{\tau}\right)\right\rangle=$ $\frac{1}{\omega} \int_{0}^{\omega} B_{i}\left(\bar{u}_{\tau}(s)\right) y^{\omega}(s) d s$, where $y^{\omega}$ is $\omega$-periodic solution that corresponds to $\omega$-periodic control input $u$. On the one hand, the functional $B_{i}$ is linear and continuous, so it is bounded and there exists a constant $M$ such that $M-B_{i}\left(\bar{u}_{\tau}(s)\right) \geq 0$ for all $s \in[0, \omega]$. On the other hand, for any $\xi, \eta \in \mathbf{L}_{[0, \omega]}^{\infty}$ we have $\xi \cdot \eta \leq\|\xi\|_{\mathbf{L}_{[0, \omega]}^{2}}\|\eta\|_{\mathbf{L}_{[0, \omega]}^{2}}$ and $\|\xi\|_{\mathbf{L}_{[0, \omega]}^{2}} \leq \frac{1}{\sqrt{\omega}}\|\xi\|_{\mathbf{L}_{[0, \omega]}^{1}}$. Hence, taking into account positivity of the system (9) we derive

$$
\begin{aligned}
\frac{1}{\omega} \int_{0}^{\omega}-B_{i}\left(\bar{u}_{\tau}(s)\right) y^{\omega}(s) d s & =-\frac{M}{\omega} \int_{0}^{\omega} y^{\omega}(s) d s+\frac{1}{\omega} \int_{0}^{\omega}\left(M-B_{i}\left(\bar{u}_{\tau}(s)\right)\right) y^{\omega}(s) d s \\
& \leq-\frac{M}{\omega} \int_{0}^{\omega} y^{\omega}(s) d s+\left(\frac{1}{\omega} \int_{0}^{\omega} M-B_{i}\left(\bar{u}_{\tau}(s)\right) d s\right)\left(\frac{1}{\omega} \int_{0}^{\omega} y^{\omega}(s) d s\right) \\
& =\left(-\frac{1}{\omega} \int_{0}^{\omega} B_{i}\left(\bar{u}_{\tau}(s)\right) d s\right)\left(\frac{1}{\omega} \int_{0}^{\omega} y^{\omega}(s) d s\right)
\end{aligned}
$$

Taking into account Lemma 5 we derive

$$
\langle u, \mathbb{1}\rangle \sum_{i=1}^{N_{2}} b_{i}=-A(\mathbb{1})\langle y, \mathbb{1}\rangle-\sum_{i=1}^{N_{2}}\left\langle y, B_{i}\left(\bar{u}_{\tau}\right)\right\rangle \leq-\left(A(\mathbb{1})+\frac{1}{\omega} \int_{0}^{\omega} \sum_{i=1}^{N_{2}} B_{i}\left(\bar{u}_{\tau}(s)\right) d s\right)\langle y, \mathbb{1}\rangle .
$$


Using the fact that $\bar{u}$ is $\omega$-periodic such that $\frac{1}{\omega} \int_{0}^{\omega} \bar{u}(s) d s=\frac{t^{\prime}}{\omega}$ and Riesz Theorem [51], we can write for some $\mu_{i} \in \mathbf{L}_{[-\tau, 0]}^{2}$ :

$$
\begin{aligned}
\frac{1}{\omega} \int_{0}^{\omega} \sum_{i=1}^{N_{2}} B_{i}\left(\bar{u}_{\tau}(s)\right) d s & =\frac{1}{\omega} \int_{0}^{\omega} \sum_{i=1}^{N_{2}} \int_{-\tau}^{0} \mu_{i}(\sigma) \bar{u}_{\tau}(s+\sigma) d \sigma d s \\
& =\sum_{i=1}^{N_{2}} \int_{-\tau}^{0} \mu_{i}(\sigma)\left(\frac{1}{\omega} \int_{0}^{\omega} \bar{u}_{\tau}(s+\sigma) d s\right) d \sigma \\
& =\sum_{i=1}^{N_{2}} \int_{-\tau}^{0} \mu_{i}(\sigma)\left(\frac{t^{\prime}}{\omega}\right) d \sigma=\frac{t^{\prime}}{\omega} \sum_{i=1}^{N_{2}} B_{i}(\mathbb{1})
\end{aligned}
$$

which completes the proof.

\subsection{Sketch of the proof of Theorem 1}

The proof is based on Fixed-Point Theorem for a generalized Banach spaces $\mathbf{B}=$ $\mathbf{L}_{[0, \omega]}^{2} \times \ldots \times \mathbf{L}_{[0, \omega]}^{2}$, which have vector-valued norms: $\llbracket x \rrbracket=\left(\left\|\boldsymbol{x}_{0}\right\|_{\mathbf{L}_{[0, \omega]}^{2}}, \ldots,\left\|\boldsymbol{x}_{n-1}\right\|_{\mathbf{L}_{[0, \omega]}^{2}}\right)^{\top} \in$ $\mathbb{R}_{+}^{n}$ for any $\boldsymbol{x} \in \mathbf{B}$. The space $\mathbb{R}_{+}^{n}$ is semi-ordered using the conventional componentwise order relation, i.e. $\boldsymbol{x} \leq \boldsymbol{y}$ if $x_{i} \leq y_{i}, i=1, \ldots, n$, where $\boldsymbol{x}, \boldsymbol{y} \in \mathbb{R}_{+}^{n}$. In this case the contraction condition of the operator $\mathcal{F}: \mathbf{B} \rightarrow \mathbf{B}$ is

$$
\llbracket \mathcal{F} \boldsymbol{x}-\mathcal{F} \boldsymbol{y} \rrbracket \leq Q \rrbracket \boldsymbol{x}-\boldsymbol{y} \rrbracket, \quad \text { for all } \mathbf{x}, \mathbf{y} \in \mathbf{B},
$$

where $Q \in \mathbb{R}_{+}^{n \times n}$ is a Schur stable matrix. Under this condition the operator $\mathcal{F}$ has the unique fixed point $\boldsymbol{x}^{*}$ on $\mathbf{B}$, i.e. $\mathcal{F}\left(\boldsymbol{x}^{*}\right)=\boldsymbol{x}^{*}$. To complete the proof of Theorem 1 let us consider the operator $\mathcal{F}=\left(\mathcal{F}_{0}, \ldots, \mathcal{F}_{n-1}\right)^{\top} \in \mathbf{B}$ with

$$
\mathcal{F}_{j}(\boldsymbol{x})=\int_{0}^{\omega} G^{(j)}(t, s) f\left(s, x_{0}(s), \ldots, x_{0}\left(s-h_{m}\right), x_{1}(s), \ldots\right) d s
$$

where $G$ is the Green function of the system $\mathcal{A} x_{h_{m}}(t)=0$. Finally, it can be shown [46] that $\mathcal{F}$ satisfies the contraction condition with the matrix

$$
Q=\left(l_{0}, \ldots, l_{n-1}\right)^{\top}\left(\sigma_{0}, \ldots, \sigma_{n-1}\right)
$$

that is Schur stable due to condition $\left(l_{0}, \ldots, l_{n-1}\right)\left(\sigma_{0}, \ldots, \sigma_{n-1}\right)^{\top}<1$.

\section{References}

[1] S. L. Brunton and B. R. Noack, "Closed-Loop Turbulence Control: Progress and Challenges," Applied Mechanics Reviews, vol. 67, no. 5, pp. 050 801-050801, Aug. 2015. [Online]. Available: http://dx.doi.org/10.1115/1.4031175

[2] M. Gad-el Hak, Flow Control: Passive, Active, and Reactive Flow Management. Cambridge CB2 2RU, UK: Cambridge University Press, 2006, google-Books-ID: 1IiFetRXG3kC.

[3] M. Palaviccini, L. Cattafesta, and B. George, "Passive Flow Control over a Three-Dimensional Turret with a Flat Aperture." American Institute of Aeronautics and Astronautics, Jun. 2011. [Online]. Available: http://arc.aiaa.org/doi/10.2514/6.2011-3265 
[4] K. B. Chun and H. J. Sung, "Control of turbulent separated flow over a backwardfacing step by local forcing," Experiments in Fluids, vol. 21, no. 6, pp. 417-426, Nov. 1996.

[5] G. V. Selby, J. C. Lin, and F. G. Howard, "Control of low-speed turbulent separated flow using jet vortex generators," Experiments in Fluids, vol. 12, no. 6, pp. 394-400, Apr. 1992.

[6] Y. Tian, Q. Song, and L. Cattafesta, "Adaptive Feedback Control of Flow Separation." American Institute of Aeronautics and Astronautics, Jun. 2006. [Online]. Available: http://arc.aiaa.org/doi/10.2514/6.2006-3016

[7] Y. Tian, L. Cattafesta, and R. Mittal, "Adaptive Control of Separated Flow." American Institute of Aeronautics and Astronautics, Jan. 2006. [Online]. Available: http://arc.aiaa.org/doi/10.2514/6.2006-1401

[8] L. N. Cattafesta III, Q. Song, D. R. Williams, C. W. Rowley, and F. S. Alvi, "Active control of flow-induced cavity oscillations," Progress in Aerospace Sciences, vol. 44, no. 7-8, pp. 479-502, Oct. 2008. [Online]. Available: //www.sciencedirect.com/science/article/pii/S0376042108000584

[9] R. J. Volino, "Separation Control on Low-Pressure Turbine Airfoils Using Synthetic Vortex Generator Jets," in ASME Turbo Expo 2003, collocated with the 2003 International Joint Power Generation Conference., Atlanta, Georgia, USA, 2003, pp. 845-859. [Online]. Available: http://dx.doi.org/10.1115/GT200338729

[10] K. McManus, H. Legner, and S. Davis, "Pulsed vortex generator jets for active control of flow separation." American Institute of Aeronautics and Astronautics, Jun. 1994. [Online]. Available: http://arc.aiaa.org/doi/10.2514/6.1994-2218

[11] R. Eldredge and J. Bons, "Active Control of a Separating Boundary Layer with Steady Vortex Generating Jets - Detailed Flow Measurements," in 42nd AIAA Aerospace Sciences Meeting and Exhibit, ser. Aerospace Sciences Meetings. Reno, Nevada, USA: American Institute of Aeronautics and Astronautics, Jan. 2004, dOI: 10.2514/6.2004-751 DOI: 10.2514/6.2004-751. [Online]. Available: http://dx.doi.org/10.2514/6.2004-751

[12] C. Raibaudo, F. Kerherve, and M. Stanislas, "Characterisation of the transient dynamics of a controlled separated flow using phase averaged PIV," in International Conference on Instability and Control of Massively Separated Flows ICOMASEF, Prato, Italy, Sep. 2013, pp. 183-188.

[13] C. Raibaudo, A. Polyakov, D. Efimov, F. Kerherve, J.-P. Richard, and M. Stanislas, "Experimental closed-loop control of a detached boundary layer at high Reynolds number," in 10th EUROMECH Fluid Mechanics Conference, Cambridge UK, Mar. 2015.

[14] T. Chabert, J. Dandois, É. Garnier, and L. Jacquin, "Experimental detection of flow separation over a plain flap by wall shear stress analysis with and without steady blowing," Comptes Rendus Mécanique, vol. 342, no. 6, pp. 389-402, 2014.

[15] T. Chabert, J. Dandois, and É. Garnier, "Experimental closed-loop control of flow separation over a plain flap using slope seeking," Experiments in Fluids, vol. 55, no. 8, pp. 1-19, 2014. 
[16] T. Shaqarin, C. Braud, S. Coudert, and M. Stanislas, "Open and closed-loop experiments to identify the separated flow dynamics of a thick turbulent boundary layer," Experiments in Fluids, vol. 54, no. 2, pp. 1-22, Jan. 2013.

[17] C. Cierpka, T. Weier, and G. Gerbeth, "Electromagnetic Control of Separated Flows Using Periodic Excitation with Different Wave Forms," in Active Flow Control, ser. Notes on Numerical Fluid Mechanics and Multidisciplinary Design (NNFM), P. D. R. King, Ed. Springer Berlin Heidelberg, 2007, no. 95, pp. $27-41$.

[18] V. Ciobaca and J. Wild, "An Overview of Recent DLR Contributions on Active Flow-Separation Control Studies for High-Lift Configurations," Aerospace Lab, vol. Issue, no. 2013-06, pp. 1-12, 2013.

[19] E. Åkervik, J. Hoepffner, U. Ehrenstein, and D. S. Henningson, "Model reduction and control of a cavity-driven separated boundary layer," in IUTAM Symposium on Flow Control and MEMS. London, England: Springer, 2008, pp. 147-155.

[20] D. Wachsmuth, "Optimal control of the unsteady Navier-Stokes equations," Ph.D. dissertation, PhD thesis, Technische Universität Berlin, 2006.

[21] O. Ghattas and J.-H. Bark, "Optimal Control of Two- and Three-Dimensional Incompressible Navier-Stokes Flows," Journal of Computational Physics, vol. 136, no. 2, pp. 231-244, Sep. 1997.

[22] E. Fernández-Cara, S. Guerrero, O. Y. Imanuvilov, and J. P. Puel, "Local exact controllability of the Navier-Stokes system," Journal de Mathématiques Pures et Appliquées, vol. 83, no. 12, pp. 1501-1542, Dec. 2004.

[23] T. Duriez, V. Parezanovic, J.-C. Laurentie, C. Fourment, J. Delville, J.-P. Bonnet, L. Cordier, B. R. Noack, M. Segond, M. W. Abel, N. Gautier, J.-L. Aider, C. Raibaudo, C. Cuvier, M. Stanislas, and S. L. Brunton, "Closed-loop control of experimental shear flows using machine learning," in 7th AIAA Flow Control Conference. Atlanta, GA, USA: American Institute of Aeronautics and Astronautics, 2014, pp. 2219-2234.

[24] M. Feingesicht, C. Raibaudo, A. Polyakov, F. Kerherve, and J.-P. Richard, "A bilinear input-output model with state-dependent delay for separated flow control," Jun. 2016. [Online]. Available: https://hal.inria.fr/hal01298166/document

[25] V. Utkin, Sliding modes in optimization and control problems. Springer Verlag, New York, 1992.

[26] C. Edwards and S. Spurgeon, Sliding Mode Control: Theory And Applications. London EC4A 3DE, UK: CRC Press, Aug. 1998, google-Books-ID: uH2RJhIPsiYC.

[27] Y. Shtessel, C. Edwards, L. Fridman, and A. Levant, Sliding mode control and observation. New York, NY 10013, USA: Springer, 2014.

[28] J. Liu, W. Luo, X. Yang, and L. Wu, "Robust Model-Based Fault Diagnosis for PEM Fuel Cell Air-Feed System," IEEE Transactions on Industrial Electronics, vol. 63, no. 5, pp. 3261-3270, May 2016. 
[29] J. Liu, S. Vazquez, L. Wu, A. Marquez, H. Gao, and L. G. Franquelo, "Extended State Observer-Based Sliding-Mode Control for Three-Phase Power Converters," IEEE Transactions on Industrial Electronics, vol. 64, no. 1, pp. 22-31, Jan. 2017.

[30] L. Fridman, P. Acosta, and A. Polyakov, "Robust eigenvalue assignment for uncertain delay control systems," in Proc. 3rd IFAC Workshop on Time Delay Systems, Santa Fe, New Mexico, USA, 2001, pp. 239-244.

[31] A. Polyakov, "Minimization of disturbances effects in time delay predictor-based sliding mode control systems," Journal of the Franklin Institute, vol. 349, no. 4, pp. 1380-1396, May 2012.

[32] L. Fridman, V. Strygin, and A. Polyakov, "Nonlocal stabilization via delayed relay control rejecting uncertainty in a time delay," International Journal of Robust and Nonlinear Control, vol. 14, no. 1, pp. 15-37, 2004.

[33] X.-G. Yan, S. K. Spurgeon, and C. Edwards, "Static output feedback sliding mode control for time-varying delay systems with time-delayed nonlinear disturbances," International Journal of Robust and Nonlinear Control, vol. 20, no. 7, pp. $777-788,2010$.

[34] J. P. Gauthier and I. Kupka, "A separation principle for bilinear systems with dissipative drift," IEEE transactions on automatic control, vol. 37, no. 12, pp. 1970-1974, 1992.

[35] E. Fridman, Introduction to Time-Delay Systems: Analysis and Control. Basel, Switzerland: Birkhäuser, Sep. 2014.

[36] C. Raibaudo, "Characterization of the transient of a separated turbulent boundary layer under control and applications to advanced closed-loop controllers," Ph.D. dissertation, Ecole Centrale de Lille, 2015.

[37] C. Raibaudo, A. Polyakov, F. Kerherve, J.-P. Richard, and M. Stanislas, "Experimental open-loop and closed-loop control of a massive separated boundary layer at high Reynolds number," Mar. 2015.

[38] C. Audet, J. E. D. Jr, and S. L. Digabel, "Globalization strategies for Mesh Adaptive Direct Search," Computational Optimization and Applications, vol. 46, no. 2, pp. 193-215, Jun. 2009.

[39] S. Le Digabel, "Algorithm 909: NOMAD: Nonlinear optimization with the MADS algorithm," ACM Transactions on Mathematical Software (TOMS), vol. 37, no. 4, pp. 1-15, 2011.

[40] C. Audet, V. Béchard, and S. L. Digabel, "Nonsmooth optimization through Mesh Adaptive Direct Search and Variable Neighborhood Search," Journal of Global Optimization, vol. 41, no. 2, pp. 299-318, Oct. 2007.

[41] C. Audet and J. Dennis, "Mesh Adaptive Direct Search Algorithms for Constrained Optimization," SIAM Journal on Optimization, vol. 17, no. 1, pp. 188217, Jan. 2006.

[42] J. Dandois, E. Garnier, and P.-Y. Pamart, "NARX modelling of unsteady separation control," Experiments in fluids, vol. 54, no. 2, pp. 1-17, 2013. 
[43] J. K. Hale, "Caratheodory Conditions," in Functional Differential Equations, ser. Applied Mathematical Sciences. Springer US, 1971, no. 3, pp. 30-31, dOI: 10.1007/978-1-4615-9968-5_7.

[44] A. Olbrot, "Control of retarded systems with function space constraints: Necessary optimality conditions," Control Cybernet, vol. 5, pp. 5-31, 1976.

[45] Z. Artstein, "Linear systems with delayed controls: A reduction," IEEE Transactions on Automatic Control, vol. 27, no. 4, pp. 869-879, Aug. 1982.

[46] L. Polyakova, "Generalized fixed-point theorems and periodic solutions of nonlinear equations," 2006.

[47] L. Kantorovich and G. Akilov, Functional Analysis. 1982. Pergamon Press, Oxford, 1982.

[48] L. Wu, W. X. Zheng, and H. Gao, "Dissipativity-Based Sliding Mode Control of Switched Stochastic Systems," IEEE Transactions on Automatic Control, vol. 58, no. 3, pp. 785-791, Mar. 2013.

[49] D. Efimov, A. Polyakov, E. Fridman, W. Perruquetti, and J.-P. Richard, "Delay-Dependent Positivity: Application to Interval Observers," Jul. 2015. [Online]. Available: https://hal.inria.fr/hal-01140336/document

[50] R. P. Agarwal, L. Berezansky, E. Braverman, and A. Domoshnitsky, Nonoscillation Theory of Functional Differential Equations with Applications. New York, NY: Springer New York, 2012.

[51] E. B. Davies, Linear Operators and their Spectra. Cambridge CB2 2RU, UK: Cambridge University Press, Apr. 2007, google-Books-ID: 6AjuCoaL34AC. 